\title{
Evolutionary history of dimethylsulfoniopropionate (DMSP) demethylation enzyme DmdA in marine bacteria
}

\author{
Laura Hernández $^{\text {Corresp., } 1}$, Alberto Vicens ${ }^{2}$, Luis E Eguiarte ${ }^{\text {Corresp., } 3}$, Valeria Souza ${ }^{3}$, Valerie De Anda ${ }^{4}$, José M \\ González ${ }^{1}$ \\ 1 Departamento de Microbiología, Universidad de La Laguna, La Laguna, Spain \\ 2 Departamento de Bioquímica, Genética e Inmunología, Universidad de Vigo, Vigo, Spain \\ 3 Instituto de Ecología, Departamento de Ecología Evolutiva, Universidad Nacional Autónoma de México, Mexico D.F., Mexico \\ 4 Marine Science Institute, Department of Marine Sciences, University of Texas Austin, Port Aransas, United States \\ Corresponding Authors: Laura Hernández, Luis E Eguiarte \\ Email address: dellia222@yahoo.es, luiseguiarte@gmail.com
}

Dimethylsulfoniopropionate (DMSP), an osmolyte produced by oceanic phytoplankton and bacteria, is primarily degraded by bacteria belonging to the Roseobacter lineage and other marine Alphaproteobacteria via DMSP-dependent demethylase A protein (DmdA). To date, the evolutionary history of DmdA gene family is unclear. Some studies indicate a common ancestry between DmdA and GcvT gene families and a co-evolution between Roseobacter and the DMSP-producing-phytoplankton around 250 million years ago (Mya). In this work, we analyzed the evolution of DmdA under three possible evolutionary scenarios: 1) a recent common ancestor of DmdA and GcvT, 2) a coevolution between Roseobacter and the DMSP-producing-phytoplankton, and 3) an enzymatic adaptation for utilizing DMSP in marine bacteria prior to Roseobacter origin. Our analyses indicate that DmdA is a new gene family originated from GcvT genes by duplication and functional divergence driven by positive selection before a coevolution between Roseobacter and phytoplankton. Our data suggest that Roseobacter acquired $d m d A$ by horizontal gene transfer prior to an environment with higher DMSP. Here, we propose that the ancestor that carried the DMSP demethylation pathway genes evolved in the Archean, and was exposed to a higher concentration of DMSP in a sulfur-rich atmosphere and anoxic ocean, compared to recent Roseobacter eco-orthologs (orthologs performing the same function under different conditions), which should be adapted to lower concentrations of DMSP. 
1 Evolutionary history of dimethylsulfoniopropionate

2 (DMSP) demethylation enzyme DmdA in marine

3 bacteria

4

5

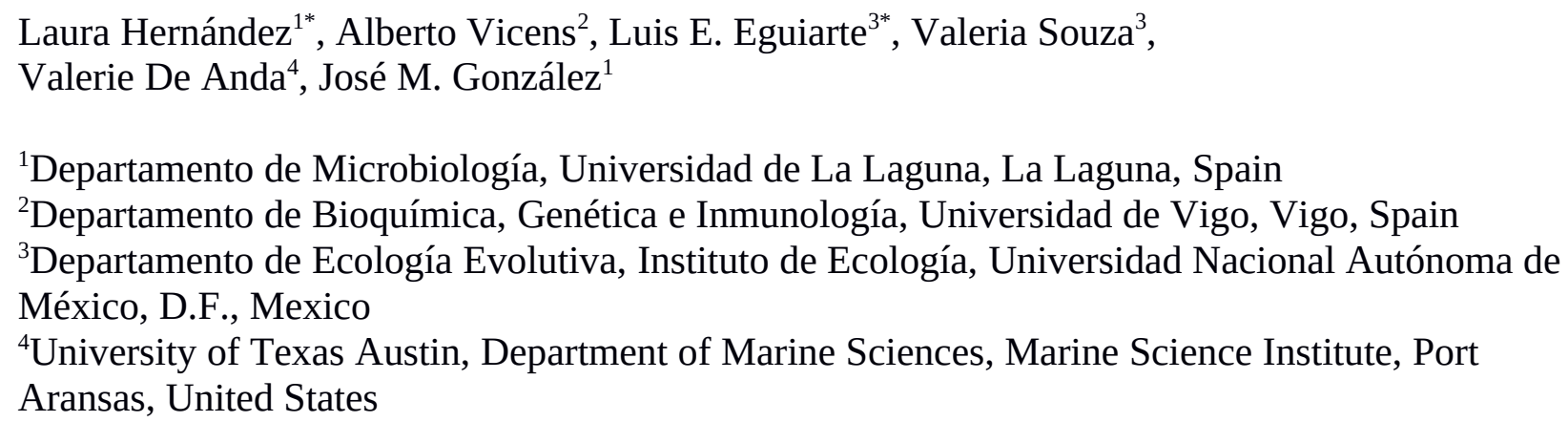

\section{Abstract}

Dimethylsulfoniopropionate (DMSP), an osmolyte produced by oceanic phytoplankton and bacteria, is primarily degraded by bacteria belonging to the Roseobacter lineage and other marine Alphaproteobacteria via DMSP-dependent demethylase A protein (DmdA). To date, the evolutionary history of DmdA gene family is unclear. Some studies indicate a common ancestry between DmdA and GcvT gene families and a co-evolution between Roseobacter and the DMSPproducing-phytoplankton around 250 million years ago (Mya). In this work, we analyzed the evolution of DmdA under three possible evolutionary scenarios: 1) a recent common ancestor of DmdA and GcvT, 2) a coevolution between Roseobacter and the DMSP-producingphytoplankton, and 3) an enzymatic adaptation for utilizing DMSP in marine bacteria prior to Roseobacter origin. Our analyses indicate that DmdA is a new gene family originated from GcvT genes by duplication and functional divergence driven by positive selection before a coevolution between Roseobacter and phytoplankton. Our data suggest that Roseobacter acquired $d m d A$ by horizontal gene transfer prior to an environment with higher DMSP. Here, we propose that the ancestor that carried the DMSP demethylation pathway genes evolved in the Archean, and was exposed to a higher concentration of DMSP in a sulfur-rich atmosphere and anoxic ocean, compared to recent Roseobacter eco-orthologs (orthologs performing the same function under different conditions), which should be adapted to lower concentrations of DMSP. 


\section{Introduction}

Dimethylsulfoniopropionate (DMSP) is an osmolyte synthesized by oceanic phytoplankton and bacteria (Galinski, 1995; Yoch, 2002; Curson et al., 2017). This molecule became abundant in the oceans approximately 250 million years ago (Mya), coinciding with the expansion and diversification of dinoflagellates (Bullock et al., 2017). Since then, it has played an important role in the biogeochemistry of sulfur cycle on Earth (Lovelock, 1983). DMSP is the main precursor of the climate-relevant gas dimethylsulfide (DMS; Reisch et al., 2011). In marine ecosystems, DMSP is rapidly degraded by different bacterial communities (González et al., 1999), and some strains seem to be very efficient and even become dependent on its degradation (Tripp et al., 2008). In fact, DMSP supports up to $13 \%$ of the bacterial carbon demand in surface waters, making it one of the most significant substrates for bacterioplankton (Kiene et al., 1999; González et al., 1999). Candidatus Pelagibacter ubique (SAR11), dominant in the bacterioplankton and especially in surface waters, can only use sulfur atoms derived organic molecules, such as DMSP (Tripp et al., 2008). In the case of Ruegeria pomeroyi DSS-3, a model organism for DMSP studies, the turnover rate of DMSP transformation depends on salinity conditions (Salgado et al., 2014).

The first step in the degradation of DMSP involves two competing pathways, cleavage and demethylation. The DMSP cleavage pathway metabolizes DMSP with the release of DMS (Kiene et al., 1999), a step catalyzed by a number of enzymes (Curson et al., 2011). In the alternative pathway, DMSP is first demethylated by a DMSP-dependent demethylase A protein (DmdA; Howard et al., 2006). Compared to genes in the DMS-releasing pathway, $d m d A$ is more frequently found in the genomes of oceanic bacteria (Newton et al., 2010; Todd et al., 1999). The DmdA enzyme was originally annotated as a glycine cleavage T-protein (GcvT) in the model bacteria $R$. pomeroyi (Reisch et al., 2011a), although it forms a separate clade from the known GcvTs (gcvT and Unchar. AMT) (Sun et al., 2011; Bullock et al., 2017). Despite their structural similarity which might indicate a common ancestry, DmdA and GcvT are mechanistically distinct (Schuller et al., 2012). DmdA produces 5-methyl-THF from DMSP as the result of a redoxneutral methyl transfer, while GcvT produces glycine to 5,10-methylene-THF from glycine (Reisch et al., 2008).

Nearly all known DMSP-catabolizing bacteria belong to the phylum Proteobacteria with DmdA orthologs found in most of the sequenced members of the Rhodobacteraceae family, as well as bacterioplankton strains of SAR11, SAR324, SAR116 and in marine Gammaproteobacteria (González et al., 1999; González, 2003; Howard et al., 2006; Bürgmann et al., 2007; Reisch et al., 2008) like Chromatiales which could have gotten DmdA gene by HGT as some studies suggest (Howard et al., 2006; González et al., 2019 ). This phylogenetic distribution suggests an expansion of $d m d A$ through HGT events between different lineages of bacteria, presumably through viruses (Raina et al., 2010). Since an episode of genome expansion of Roseobacter, predicted early in its genome evolution, coincides with the diversification of the dinoflagellates and coccolithophores around 250 Mya (Luo et al., 2013; Luo \& Moran, 2014), it has been suggested a co-evolutionary event between Roseobacter and the DMSP-producing-phytoplankton (Luo et al., 2013; Luo \& Moran, 2014; Bullock et al., 2017). Under this scenario, the enzymes of the DMSP demethylation pathway could have evolved within the last 250 Mya, as phytoplankton responded to the marine catastrophe at the end of the Permian, with the diversification of dinoflagellates that produce DMSP and the Roseobacter clade expanding by using DMSP as its main sulfur source. Despite this hypothesis, there is a lack of knowledge about the main evolutionary events that lead the adaptation to DMSP in Roseobacter. 
97 The biosynthesis of DMSP has been reported in marine heterotrophic bacteria, such as the

98 Alphaproteobacteria, i.e. Labrenzia aggregata (Curson et al., 2017), Gammaproteobacteria and

99 Actinobacteria (Williams et al., 2019). Moreover, bacteria seem to be important producers of

100 DMSP and DMS in coastal and marine sediments (Williams et al., 2019). Since the common

101 ancestor of heterotrophic bacteria and Roseobacter originated in the Archean, more than 2 billion

102 years ago (Kumar et al., 2017), the Roseobacter and other Alphaproteobacteria might have been

103 exposed to DMSP early (Reisch et al. 2011a,b). According to this hypothesis, the DMSP

104 demethylation and the cleavage pathways arose by the evolution of enzymes that were already

105 present in bacterial genomes and adapted in response to the wide availability of DMSP. As

106 mentioned earlier, Alphaproteobacteria in the SAR11 group seems to thrive at the expense of

107 organic sulfur compounds, such as DMSP, and had a common ancestor that lived ca. 826 Mya, at

108 the end of the Precambrian (Luo et al., 2013). We would then expect a common ancestor of the

109 DmdA gene family during the early Proterozoic and that the functional divergence between

110 DmdA and GcvT gene families was driven by both functional constraints and widespread HGT,

111 probably during the Huronian snowball Earth, a period of planetary crisis where the greatest

112 microbial diversity took refuge in the shallow seas close to the equator (Tang, Thomas, \& Xia, 113 n.d.).

114

Here, we analyzed the evolutionary history of the DmdA gene family in marine Proteobacteria by considering three evolutionary scenarios: 1) a recent common ancestry of DmdA and GcvT, 2) a coevolution between Roseobacter and the DMSP-producing-phytoplankton, and 3) an enzymatic adaptation for utilizing DMSP in marine bacteria prior to Roseobacter origin. We first analyzed if convergent, independent or HGT-based evolution can explain the presence of $d m d A$ genes in different bacterial lineages SAR11, SAR116 and Rhodobacteraceae. Then, we inferred the most recent common ancestor (MRCA) of the DmdA gene family, the timing of its origin and any duplication events. We also reconstructed the ancestral forms of DmdA enzymes to infer the most likely ecological conditions where DmdA thrive. We provide insights into their function by analyzing DmdA structural evolution. Finally, we examined how natural selection could have driven the divergence of the DmdA gene family. Our results indicate that $d m d A$ appeared before the origin of the Roseobacter clade and the conditions of the late Permian created by eukaryotic phytoplankton. Therefore, DmdA is an adapted version of enzyme that evolved in response to the availability of DMSP.

\section{Materials \& Methods}

\section{Data mining}

Peptides and genes from DmdA gene family were collected from a set of 771 genomes manually curated and hosted in the MarRef database (Klemetsen et al., 2018). The DmdA orthologs and homologs sequences were obtained as described by González et al. (2019). The DmdA homologs included were obtained using a HMM designed for DmdA orthologs (González et al., 2019), with a relaxed maximum e-value (e-50). A total of 204 sequences from 184 genomes were used to infer the evolutionary history of DmdA gene family (Table S1).

\section{Phylogenetic tree reconstruction and topology tests}

The phylogenetic tree of the DmdA protein sequences included DmdA orthologs and DmdA homologs (non-DmdA as in González et al (2019)). The sequences were aligned using MUSCLE (Edgar, 2004). Regions poorly aligned or with gaps were removed using TrimAl (CapellaGutiérrez et al., 2009) with parameters set to a minimum overlap of 0.55 and a percent of good 
146

147

148

149

150

151

152

153

154

155

156

157

158

159

160

161

162

163

164

165

166

167

168

169

170

171

172

173

174

175

176

177

178

179

180

181

182

183

184

185

186

187

188

189

190

191

192

positions to 60. Best-fit evolutionary model was selected based on the results of the package ProtTest 3 (Darriba et al., 2011) to determine the best-fit model for maximum likelihood (ML) and Bayesian inference (BI).

For the maximum likelihood analysis (ML), PhyML v3.0 (Guindon et al., 2010) or RAxML v7.2.6 (Stamatakis, 2006) were used to generate 100 ML bootstrap trees, using the Le Gascuel (LG; Le \& Gascuel, 2008) model with a discrete gamma distribution $(+G)$ with four rate categories, as this was the model with the lowest Akaike information criterion and Bayesian information criterion score. For the Bayesian analysis (BI), trees were constructed using the PhyloBayes program (Lartillot \& Philippe, 2004, 2006; Lartillot et al., 2007) with the CAT model that integrates heterogeneity of amino acid composition across sites of a protein alignment. In this case, two chains were run in parallel and checked for convergence using the tracecomp and bpcomp scripts provided in PhyloBayes. As an alternative, we computed a phylogenetic tree using a BI implemented in BEAST2 program which was run with relaxed clock model and Birth Death tree prior (Bouckaert et al., 2014). Finally, we used R v3.6.1 (R Core Team, 2017) with phangorn v2.5.5 (Schliep, 2011) to perform consensus unrooted trees.

We ran several topology tests to establish whether the trees generated using the ML and BI methods provided an equivalent explanation for the two main groups, i.e., the non-DmdA and DmdA clades. For this analysis, the topologies were compared with the TOPD/FMTS software v4.6 (Puigbo et al., 2007). A random average split distance of 100 trees was also created to check if the differences observed were more likely to have been generated by chance.

\section{Horizontal gene transfer (HGT) test and GC content analysis}

Two approaches were used to detect HGT. First, a phylogenetic incongruence analysis (Ravenhall et al., 2015) through three topology tests, the Kishino-Hasegawa (KH) (Kishino \& Hasegawa, 1989), the Shimodaira-Hasewaga (SH) (Shimodaira \& Hasegawa, 1999) and the approximately unbiased (AU) (Shimodaira, 2002), implemented in the IQ-TREE software v1.5.5 (Nguyen et al., 2015). Two topologies were tested, the ML topology obtained for the species tree of the genomes here analyzed, and the ML phylogeny of DmdA. To construct the species tree, ribosomal protein 16 small subunit (RPS16) sequences were collected from the MarRef database (Klemetsen et al., 2018), one for each genome (Table S1).

The GC content variation was studied to identify genes that have a different percentage of GC content at the third position of codons with respect to the neighboring genomic regions. The EPIC-CoGe browser (Nelson et al., 2018) was used to visualize the genomes and sequences and look for genes that use different codons with respect to the rest of the genomic dataset (data are available under permission as "ULL-microevolution" on https://genomevolution.org/).

\section{Molecular dating}

We first tested for heterogeneities in the substitution rates of the genes using a likelihood ratio test (LRT) (Felsenstein, 1981) with the ML-inferred tree. Likelihoods' values were estimated using baseml in PAML v4.8 (Yang, 2007) under rate constant and rate variable models and used to compute the likelihood ratio test (LRT) statistic according to the following equation:

$$
\mathrm{LRT}=-2\left(\log \mathrm{L}_{1}-\log \mathrm{L}_{0}\right)
$$


193

194

195

196

197

198

199

200

201

202

203

204

205

206

207

208

209

210

211

212

213

214

215

216

217

218

219

220

221

222

223

224

225

226

227

228

229

230

231

232

233

234

235

236

237

238

239

240

241 where $L_{1}$ is the unconstrained (nonclock) likelihood value, and $L_{0}$ is the likelihood value obtained under the rate constancy assumption. LRT is distributed approximately as a chi-square random variable with (m-2) degrees of freedom (df), $m$ being the number of branches/parameters.

To conduct a molecular dating analysis with BEAST 2 (Bouckaert et al., 2014), two independent MCMC tree searches were run for 50 million generations, with a sampling frequency of 1000 generations over codon alignment obtained, as we explain in the next section. The GTR substitution model with a gamma shape parameter and a proportion of invariants (GTR + G + I), was selected with PartitionFinder software v2.1.1 (Lanfear et al., 2016) based on the Bayesian Information Criterion (Darriba et al., 2012), applied with a Birth Death tree prior (Gernhard, 2008) and an uncorrelated relaxed clock log-normal. The molecular clock was calibrated using information from the TimeTree database (Hedges et al., 2006, 2015; Kumar et al., 2017). We used the proposed dates of the most recent common ancestor of (1) the Alpha- and Gammaproteobacteria (2480 Mya), (2) the Halobacteriales (455 Mya) (Fig. S1-S3) (Hedges et al., 2006, 2015; Kumar et al., 2017), and (3) the SAR11 (826 Mya) (Luo et al., 2013). A lognormal prior distribution on the calibrated nodes centered at the values mentioned above was specified with 20 standard deviations and constrained to be monophyletic. Convergence of the stationary distribution was checked by visual inspection of plotted posterior estimates in Tracer v1.6 (Rambaut, \& Drummond, 2013) to ensure effective sample sizes (ESSs) of parameters were >> 200, as recommended by the authors. After discarding the first $15 \%$ trees as burn-in, the samples were summarized in the maximum clade credibility tree using TreeAnnotator v1.6.1 (Rambaut, \& Drummond, 2002) with a PP limit of 0.5 and summarizing mean node heights. Means and $95 \%$ higher posterior densities (HPDs) of age estimates are obtained from the combined outputs using Tracer v1.6. The results were visualized using FigTree v.1.4.3 (Rambaut, 2009).

\section{Maximum likelihood tests of positive selection}

To measure the strength and mode of natural selection during the evolution of DmdA gene family, the ratio of non-synonymous ( $\mathrm{dN})$ to synonymous substitutions (dS) $(\omega=\mathrm{dN} / \mathrm{dS})$ was calculated in CodeML implemented in the suite Phylogenetic Analysis by Maximum Likelihood (PAML package v4.8) (Yang, 2007).

CodeML requires an alignment of coding sequences, and a phylogenetic tree. DNA alignment was achieved by MUSCLE (Edgar, 2004) implemented in MEGA-CC v7.0.26 (Kumar et al., 2016) and poorly aligned segments were eliminated with Gblocks under defaults parameters (Castresana, 2000). The phylogenetic tree was built using ML with PhyML v3.0 (Guindon et al., 2010) as described above and a nucleotide substitution model selected by jModelTest (Darriba et al., 2012). DAMBE (Xia, 2001) was also used to check for saturation of nucleotide substitutions using a plot of the number of transitions and transversions for each pairwise comparison against the genetic distance calculated with the F84 model of nucleotide substitution (Huelsenbeck \& Rannala, 1997), which allows different equilibrium nucleotide frequencies and a transition ratetransversion rate bias. Multiple sequence alignments with similar characteristics (i.e., showing saturation of nucleotide substitutions) were then analyzed with CodeML (Yang, 2007).

Three sets of models were used (site-specific, branch-specific and branch-site models) to detect pervasive and episodic selection during the evolution of $d m d A$ orthologs. Likelihood-ratio tests (LRTs) were used to compare models, and significant results (p-value $<0.05$ ) were determined contrasting with a chi-square distribution (chisq) (Anisimova et al., 2001). 
242

243

244

245

246

247

248

249

250

251

252

253

254

255

256

257

258

259

260

261

262

263

264

265

266

267

268

269

270

271

272

273

274

275

276

277

278

279

280

281

282

283

284

285

286

287

288

289

In the site-specific analysis, we tested for variability of selection (type and magnitude) across the codons of the gene using three pairs of nested models. The first pair includes M0 (just one $\mathrm{dN} / \mathrm{dS}$ ratio) and M3 ("K" discrete categories of $\mathrm{dN} / \mathrm{dS}$ ) and has four degrees of freedom (df). The second pair of models considers M1a (just two classes of sites, purifying [dN/dS $<1]$ and neutral selection [dN/dS=1]) and M2a (the same as M1a adding a third class of sites dedicated to positive selection $[\mathrm{dN} / \mathrm{dS}>1]$ ), this has two df. Finally, the third pair of models comprised M7 (a beta distribution that allows $\mathrm{dN} / \mathrm{dS}$ to vary among the interval $[0,1]$ ) and M8 (adds an extra discrete category to M7 with dN/dS>1), with two df. Whereas M0 vs M3 tests for evidence of dN/dS variation across sites, M1a vs M2a and M7 vs M8 tests for the presence of sites under positive selection (dN/dS > 1).

Using three branch models (Yang, 1998), we tested for variation of selection over evolutionary time. The null model (M0) assumes that all branches evolve at the same rate, therefore, there is only one value of $\mathrm{dN} / \mathrm{dS}$ for all the branches of the tree. The two-ratio model allows two $\mathrm{dN} / \mathrm{dS}$ values, one value for all the Roseobacter lineage (we called this group A) and another for the rest of branches (group B). The free-ratio model, allows one $\mathrm{dN} / \mathrm{dS}$ value for each branch. Null and two-ratio model are compared by LRT with one df but null and free-ratio model are compared with $36 \mathrm{df}$.

For the last set of models, we identified sites that have been under positive selection at a particular point of evolution using branch-site models, in which $\mathrm{dN} / \mathrm{dS}$ can vary among sites and among branches (Zhang, 2005). We computed two models: a null model, in which the "foreground branch" may have different proportions of sites under neutral selection to the "background branches", and an alternative model in which the "foreground branch" may have a proportion of sites under positive selection. We compare these models for each terminal branch with a LRT of one df. For each branch-site analysis, we applied the Bonferroni correction for multiple testing.

In site and branch-site tests, we identified sites under positive selection as those with Bayes Empirical Bayes (BEB) posterior probability above 0.95 (Yang, 2005). We also checked for convergence of the parameter estimates in PAML by carrying out at least two runs for each tree and starting the analysis with different $\omega(0.2,1,1.2$ and 2$)$. In addition, to test for convergent selection in several lineages, we ran at branch-site analysis selecting as "foreground branches" all those under positive selection in a previous analysis.

\section{Analysis of functional divergence}

Divergent selection is indicated by different $\omega$ values among paralogous clades. We tested whether selective pressures diverged following duplication that led to $d m d A$ and non-dmdA genes (Bielawski \& Yang, 2004). We compared the M3 model, which accounts for $\omega$ variation among sites but not among branches or clades, with a model allowing a fraction of sites to have different $\omega$ between two clades of a phylogeny (clade model D). We also tested M0 and M3 models and we used a posterior BEB probability above 0.95 to identify sites evolving under divergent selective pressures. We checked for convergence of the parameter estimates in PAML by carrying out at least two runs for the tree and starting the analysis with different $\omega(0.1,0.25,2,3$ and 4).

Peer) reviewing PDF | (2019:12:44271:1:1:NEW 6 Aug 2020) 
290 Finally, we applied two branch-site models (as described above) to test dN/dS differences on the 291 branches representing the ancestral lineages of the DmdA and non-DmdA clades (see results).

292 We considered the ancestral sequences from DmdA and non-DmdA clades as foreground

293 branches in two different models.

294

295

296

297

298

299

300

301

302

303

304

305

306

307

308

309

310

311

312

313

314

315

316

317

318

319

320

321

322

323

324

325

326

327

328

329

330

331

332

333

334

335

336

337

\section{Reconstruction of ancestral DmdA sequence}

To reconstruct the ancient conditions where $d m d A$ gene prospered, we inferred the ancestral sequences of the DmdA node using the FastML web server (Ashkenazy et al., 2012) and then computed estimated physico-chemical properties on predecessor sequence using Compute ProtParam tool from Expasy - SIB Bioinformatics Resource Portal (Gasteiger et al., 2005). Moreover, we also reconstructed the ancestral sequence of the non-DmdA node, as well as the ancestral sequence of both the DmdA, and the non-DmdA families. FastML was run considering the alignment of proteins and the ML phylogenetic tree for those DmdA orthologs or homologs inferred as we explained above. Posterior amino acid probabilities at each site were calculated using the LG matrix and Gamma distribution. Both marginal and joint probability reconstructions were performed. Protein sequences resulting from marginal reconstructions were used to predict tertiary structure (see below) as well as to identify family domains using Pfam v32 (Finn et al., 2010).

\section{Protein tertiary structure analysis}

Predicted three-dimensional structures of protein sequences were examined by Iterative Threading ASSEmbly Refinement (I-TASSER) (Roy et al., 2010; Yang et al., 2015). First, ITASSER uses local meta-threading-server (LOMETS) (Wu \& Zhang, 2007) to identify templates for the query sequence in a non-redundant Protein Data Bank (PDB) structure library. Then, the top-ranked template hits obtained are selected for the 3D model simulations. To evaluate positively the global accuracy of the predicted model, a C-score should return between -5 and 2 . At the end, the top 10 structural analogs of the predicted model close to the target in the PDB (Berman et al., 2000) are generated using TM-align (Zhang, 2005). The TM-score value scales the structural similarity between two proteins and should return 1 if a perfect match between two structures is found. A TM-score value higher than 0.5 suggests that the proteins belong to the same fold family.

We used PyMol v1.7.4 (DeLano, 2002) to visualize the 3D structure of the proteins and to map the positively selected sites onto the 3D structure of DmdA (pdb: 3tfh).

\section{Results}

\section{Phylogenetic tree for DmdA family}

We identified a total of 204 DmdA protein sequences out of 150 curated genomes (see Table S1: Genomes and genomic diversity sheets), and reconstructed their evolutionary relationships using BI (Fig. 1) and ML (Fig. S4). Unrooted trees in TOPD-FMTS showed that split distances did not exceed 0.19 , indicating that the phylogenetic reconstruction is robust, with minor variations in alignment filtering and methods for inferring topologies (Table S2).

The BI tree (Fig. 1) shows a main duplication between two lineages. The larger phylogenetic group comprises genes mainly from Bacteroidetes, while the smaller group includes genes 
338

339

340

341

342

343

344

345

346

347

348

349

350

351

352

353

354

355

356

357

358

359

360

361

362

363

364

365

366

367

368

369

370

371

372

373

374

375

376

377

378

379

380

381

382

383

384

385

386

mainly from Alphaproteobacteria. We focused on this smaller group as it includes the DmdA sequences (Fig. 1; green color) and the closest homologs to DmdA (Fig. 1; brown color).

Using phylogenetic analyses including DmdA orthologs and DmdA homologs close to those (the limit to select the closer homologs was set to a maximum e-value of e-80), we resolve the position of the first DmdA sequences isolated from two marine bacterial species, $R$. pomeroyi (AAV95190.1) and Ca. P. ubique (AAZ21068.1). In addition, the inclusion of DmdA homologs allowed to resolve a robust phylogenetic relationship of the DmdA gene family (Fig. 2). We detected a clear separation between DmdA and putative non-DmdA families. Indeed, the four DmdA family trees constructed using different methods compared in TOPD-FMTS using split distances (Table S3) and unrooted trees (Fig. S5) agreed with this result. The average split distance was 0.60 , indicating that the trees were neither identical (split difference $=0$ ) nor completely different (1). A random split distance was calculated to analyze whether the split distances were significantly different. Because the random split distance resulted in a value close to 1 (0.988), our observations are unlikely to be given by chance.

To identify HGT and duplication events, we constructed a proxy for the species tree of the genomes considered here by using a set of small subunit ribosomal protein (see Material and Methods). Given this (proxy) species tree (Fig. S6), the positions of many sequences on the DmdA tree are better explained as cases of HGT (Fig. S6; Fig. 3) with high statistical support. Then we tested whether the topology for a common set of taxa within the DmdA family (Fig. S7) similar to that of the species tree (Fig. S8). We found significant differences (at an alpha of 0.01) between the topology of DmdA group and that of the proxy species tree (Table S4); this incongruence between phylogenies is conserved irrespective of the test used (KH, SH and unbiased tests). From these results, we concluded that the phylogenetic relationships within each DmdA group were different to those of the species tree, strongly supporting a HGT-based evolution of DmdA family (Fig. S8). Moreover, we found many genes that use different codons from the neighboring genomic regions. These genes are inferred as having been horizontally transferred given their $(\mathrm{G}+\mathrm{C})$ wobble content (Table $\mathrm{S} 1)$, supporting HGT as a plausible mechanism of genomic variability which introduces more variation than vertical gene transfer (VGT) and that contribute to DmdA evolution (Fig. S8).

\section{Structural modeling}

The structure for DmdA orthologs inferred on the protein sequences by Iterative Threading ASSEmbly Refinement (I-TASSER) were threaded onto the known structure of DMSPdependent demethylase A protein (PDB accession: 3tfhA) with a C-score $<=2$ (Table S5). However, the predicted models for DmdA homologs were threaded onto two types of known structure; DmdA orthologs, and the structure of the mature form of rat dimethylglycine dehydrogenase (DmgdH) (PDB accession, 4ps9sA) with a C-score $<2$ except for the sequence with accession number AEM59334.1, which showed a C-score > 2 (Fig. S9a, Fig. S9b, Data S1).

We clustered sequences with a putative DmgdH structure in a separate group using principal component analysis (Fig. S9c). There is a clear 3D-structure coincidence between DmdA clade (green color in Fig. S9a) and the majority of lineages from non-DmdA clade (brown color in Fig. S9a), as well as a conserved folate-binding domain (Fig. S9b: 99S, 178E and 180Y). However, in the alignment we found a pattern of conserved residues coherent with the phylogenetic results (Fig. S9a, Fig. S9b), where non-DmdA clade is formed by three subclades, one of them with DmgdH tertiary structure. Indeed, a key residue for DMSP specific interaction is shown in clades

PeerJ reviewing PDF | (2019:12:44271:1:1:NEW 6 Aug 2020) 
387

388

389

390

391

392

393

394

395

396

397

398

399

400

401

402

403

404

405

406

407

408

409

410

411

412

413

414

415

416

417

418

419

420

421

422

423

424

425

426

427

428

429

430

431

432

433

434

435

with DmdA tertiary structure (Fig. S9b: W171), but not in a clade with DmgdH tertiary structure (Fig. S9b: F171).

\section{Molecular dating}

The log likelihood test (LRT) detected heterogeneity in the substitution rates of $d m d A$ orthologs and $d m d A$ homologs genes (Fig. 2) ( $\log \mathrm{L}_{0}=-29,827.108$; $\log \mathrm{L}_{1}=-29,546.053$; degrees of freedom $=46$; chisq $=562.11 ; \mathrm{P}<0.001$ ), thus rejecting the hypothesis of a strict molecular clock. This finding validates the use of a relaxed molecular clock approach to estimate the node ages through Bayesian analysis (see Methods for details). We observed that the marginal densities for each run of the divergence time estimate analysis were nearly identical, pointing that the runs converged on the same stationary distributions. In all runs the marginal densities for the standard deviation hyperparameter of the uncorrelated log-normal relaxed clock model were quite different from the prior, with no significant density at zero, and with a coefficient of variation around 0.2. Analyses using three different calibrated prior dates showed no discrepancies in the final divergence time estimates (Table S6).

The time estimates for the MRCA of each gene family (Table S6 and Fig. 4) indicate that the most recent common ancestor of DmdA gene family occurred in the late Archean, around 2,400 Mya, after a gene duplication event. Also, a duplication within the DmdA lineage generated a separated SAR11 and Roseobacter DmdA lineage in the early Precambrian ca. 1,894 Mya (Fig. 4: red arrow). Ca. P. ubique HTCC1062 within the SAR11 cluster and $R$. pomeroyi DSS-3 within the Roseobacter cluster, resulted from a duplication around 300 Mya (Fig. 4: blue arrow). However, a higher number of duplication events took place in the second cluster. (Fig. 4: green color).

We detected two duplication events within the putative non-DmdA clade (Fig. 4; brown color); showing that the gene families were originated through old duplication events. One duplication involving the DmgdH family (Fig. 4: light yellow color; Table S5) occurred ca. 1,480 Mya and another duplication ca. 1,000 Mya (Fig. 4: green arrow), involving a gene family with tertiary structure similar to $\mathrm{Ca}$. P. ubique DmdA The other duplication event took place during the Huronian glaciation, around 2100 Mya (Fig. 4: violet arrow).

\section{Reconstruction of ancestral DmdA sequence}

Our analysis was focused on the reconstruction of the ancestral sequences of the DmdA clade, the non-DmdA clade as well as the ancestral sequence of both the DmdA and non-DmdA clades. FastML inferred the 100 most likely ancestral sequences of the DmdA family. We observed that the same sequences were always inferred. Indeed, the difference in log-likelihood between the most likely ancestral sequence at this node (N1; Fig. S10) and the 100th most likely sequence was only 0.105 , indicating that both sequences were almost as likely to reflect the "true" ancestral sequence. That ancestral protein contains both PF01571 (GCV_T) and PF08669 (GCV_T_C) domains, found in the DmdA orthologs and it is nearly identical to Ca. P. ubique HTCC1062 DmdA sequence. Moreover, PSI-BLAST search confirmed that the ancestral sequence in node 1 close to DmdA genes hosted in EMBL-EBI databases (Fig. S11) and the structure for Ca. P. ubique apoenzyme DmdA was the closest analog to our predicted models (Table S5; Data S1). Inferred physico-chemical properties are identical between $\mathrm{Ca}$. P. ubique and the DmdA ancestral sequence (Table S7). 
436

437

438

439

440

441

442

443

444

445

446

447

448

449

450

451

452

453

454

455

456

457

458

459

460

461

462

463

464

465

466

467

468

469

470

471

472

473

474

475

476

477

478

479

480

481

482

483

On the other hand, the ancestral sequence inferred for non-DmdA family (N1; Fig. S12) and the ancestral sequence previous to functional divergence (N1; Fig. S13) contains only the PF01571 domain. That domain was located onto the known structure of T-protein of the Glycine Cleavage System (PDB accession: 1wooA) with a C-score= 1.25 (Table S5; Data S1) in the case of the ancestral DmdA and non-DmdA sequence. However, the ancestral sequence for non-DmdA was better threaded onto the known structure of mature form of rat DmgdH (PDB accession: 4p9sA) with a C-score= 0.76 (Table S5; Data S1).

\section{Detection of positive selection on $\mathrm{dmdA}$ sequences}

To infer how natural selection has influenced the evolutionary history of DmdA gene family, we used an alignment of the 20 sequences clustered as $d m d A$ orthologs (Fig. S14). The phylogenetic tree for these sequences was constructed by ML using the symmetrical model (SYM) with a discrete gamma distribution.

The average $\mathrm{dN} / \mathrm{dS}$ value for the $d m d A$ gene was 0.085 , suggesting that this gene evolved under strong negative (purifying) selection. Then, we analyzed $\mathrm{dN} / \mathrm{dS}$ variation across the codons in the gene, comparing M0 and M3 models through a LRT. The M3 model fits the data better than the M0 model (chisq= 775.387, p-value $<0.01$ ). All codons in the gene are under strong purifying selection with $\mathrm{dN} / \mathrm{dS}<1$ (Fig. 5), which indicates that this sulfur pathway is important for the cells. In accordance with this, the LTRs designed to detect codons under positive selection were not significant (M1 vs M2, chisq= 0 and p-value $=1$, and M7 vs M8, chisq $=1.459$ and $\mathrm{p}$-value = 0.482). Hence, we did not detect sites in $d m d A$ subjected to positive selection (Fig. S15).

We tested the variation in the intensity of selection over evolutionary time. A two-ratio model comparing the Roseobacter with the rest of lineages (Fig. S16) fits the data, as the LRT was 23.777 and p-value $<0.01$ (Table S8). dN/dS value in Roseobacter $\left(\omega_{1}: 0.0767\right)$ was significantly lower than in the remaining branches $\left(\omega_{2}: 0.1494\right)$, suggesting stronger purifying selection on $d m d A$ in Roseobacter. When we tested the intensity of selection over evolutionary time using the free-ratio model (Table S8), we found changes in the selection pressure from the branches which defines the separation of SAR11 from Roseobacter DmdA gene families (Fig. S17: branches from nodes 21 to 23). In particular, we observed a dN/dS value $>1$ in the branch connecting nodes 21-23. We also identified some more recent branches (connecting nodes 25-26 and 28-29) for which $\mathrm{dN} / \mathrm{dS}>>1$ was estimated (Fig. S17).

Finally, we applied the two branch-site models to test for sites under selection on the individual lineages associated with $d m d A$ (Fig. S18). Four sequences (WP_047029467, AHM05061.1, ABV94056.1, AFS48343.1) had a significant LRT after correcting for multiple testing (Table S9), corresponding to episodic positive selection on these lineages (Fig. S18). It should be highlighted that three selected sites are shared by at least two lineages (Table S9; Fig. 6). One shared site is located next to the GcvT domain (152 K; Fig. S19), and two shared sites are close to conserved positions (17E; 87Y; Fig. S19). The residue $87 \mathrm{Y}$ is adjacent to the conserved interaction site with THF (88Y; Fig. S19). Interestingly, since the selected lineages are separated in the tree, the adaptive mutations seem to have occurred through three parallel independent changes (Fig. S20).

\section{Functional divergence during the molecular evolution of DmdA sequences}

Peer) reviewing PDF | (2019:12:44271:1:1:NEW 6 Aug 2020) 
484

485

486

487

488

489

490

491

492

493

494

495

496

497

498

499

500

501

502

503

504

505

506

507

508

509

510

511

512

513

514

515

516

517

518

519

520

521

522

523

524

525

526

527

528

529

530

531

532

We tested whether DmdA and non-DmdA gene families were subject to different functional constrains after gene duplication (Fig. S5). We estimated the one-ratio model (M0) that yielded a value $\omega=0.053$ (Table S10), indicating that purifying selection dominated the evolution of these proteins. The discrete model (M3) was applied to these sequences (Table S10) and the LRTs comparing M0 and M3 indicated significant variation in selective pressure among sites (Table S10; Fig. S21).

The M3 model was compared with Model D, which accommodates both heterogeneity among sites and divergent selective pressures. The LRT was significant and supported the model D (Table S10), implying statistical evidence for functional divergence between DmdA and nonDmdA. Parameter estimates under Model D with $\mathrm{k}=3$ site classes suggested that $23.6 \%$ of sites were evolving under strong purifying selection $(\omega=0.006)$, while $26.7 \%$ of sites were evolving under weaker selective pressure $(\omega=0.04)$. Interestingly, a large set of sites $(49.6 \%)$ were evolving under divergent selective pressures, with weaker purifying selection in the DmdA-clade ( $\omega=0.169)$ than non-DmdA-clade $(\omega=0.100)$. We identified 77 sites evolving under divergent selective pressures between DmdA and non-DmdA (Table S10). Nineteen sites were located within the alpha helix (red tube in Fig. S22) of the secondary structure prediction and sixteen were located in the beta sheet (green arrows in Fig. S22). According to the global dN/dS estimates, for all divergent positions, $d m d A$ sequences seem to be more conserved than non$d m d A$ sequences. Moreover, this data were only compatible with recombination breaking linkage disequilibrium within the gene set that we observed with the HGT analysis.

Finally, we were interested in finding out if adaptive evolution has occurred in the lineages immediately following the main duplication event (Fig. S23). We applied two branch-site models to test for sites under selection on the ancestor associated with the DmdA and non-DmdA clades (Table S9). The LRT was significant for both ancestral branches (LRT > 7 and p-value < 0.05). Nonetheless, the foreground $\omega$ for class 2 sites tended to infinity $(\omega=999)$ in both cases, indicating lack of synonymous substitutions $(\mathrm{dS}=0)$ in these sites. We also performed two-ratio models to estimate global $\omega$ on these branches, but both estimates tended to infinity (Table S11), suggesting lack of synonymous substitution in the divergence of DmdA and non-DmdA ancestors. Therefore, although the fixation of only non-synonymous substitutions following gene duplication might indicate strong positive selection driving functional divergence of DmdA and non-DmdA families, we cannot confirm it with the applied tests.

\section{Discussion}

In this study we evaluated three scenarios for the evolutionary history of the DmdA gene family in marine bacteria. The results for each one are discussed separately.

\section{First scenario: a recent common ancestry between DmdA and GcvT}

In relation to the first scenario, we found that contrary to our initial expectations, DmdA and GcvT do not seem to have a recent common ancestry, but DmdA and non-DmdA. The clear separation between DmdA and putative non-DmdA gene families that originated in the Archean ca. 2,400 Mya after a gene duplication, supports a common recent ancestry for DmdA (Fig. 7.A) and non-DmdA (Fig. 7.B). Our tertiary structure analyses indicate that they share a putative GcvT protein as their ancestor sequence (EC 2.1.2.10). Indeed, our results agree with other studies in the case of DmdA (Reisch et al., 2008). Then, this clade seems to have been originally a GcvT (Fig. 7), as Bullock et al. (2017) suggested. 
533 The DmdA clade is a member of aminomethyltransferase (AMT/GCV_T) family with DMSP-

534 dependent demethylase tertiary structure, while non-DmdA clade includes an ancestor with a

535 tertiary structure that better matches the dimethylglycine dehydrogenase oxidorreductase

536 (DmgdH, EC 1.5.99.2) (Fig. 7.B) and members with DmdA tertiary structure. To establish

537 structural convergence as the reason of this DmdA structure coincidence between DmdA and

538 non-DmdA members, we used a phylogenetic approach based on reconstructing ancestral

539 sequences of the two clades, and then to model the ancestral proteins. We determined different

540 structural features between ancestral sequence reconstructed from DmdA and non-DmdA

541 families. In the first case, the ancestral sequence reconstructed coincides with a DmdA tertiary

542 structure (Fig. 7.A), as well as with a DmdA sequence with physico-chemical properties inferred

543 in this study (Table S7) and agree with previous ones (Reisch et al., 2008). However, the non-

544 DmdA ancestral sequence reconstructed is a DmgdH that seems to be kept in the clade called

545 DmgdH (Fig. 7. B), as well as in some members of DmdA clades (DmdA_1 and DmdA_2 within

546 non-DmdA clade) where the majority of sequence gained DmdA structure (Fig. 7.B). Therefore,

547 DmdA structural features seem to have emerged independently in both clades: DmdA and non-

548 DmdA. This finding is interesting, since known cases of structural convergence of proteins are

549 rare (Zakon, 2002). Experimental assays expressing and screening the activity of the ancestral

550 proteins at different conditions will be required to corroborate the structural convergence.

551

552

553

554

555

556

557

558

559

560

561

562

563

564

565

566

567

568

569

570

571

572

573

574

575

576

577

578

579

580

581

Since GcvT does not share the most recent common ancestry with DmdA (as we observe in Fig. 7), we examined the functional divergence between DmdA and non-DmdA clades to explain how natural selection could have driven the divergence of the DmdA gene family. We found 77 codon sites evolving under divergent selective pressures between DmdA and non-DmdA gene families. Structural divergence seemed to be imposed on the protein during sequence divergence, since 19 sites were located within the alpha helix of 2D structure and 16 in the beta sheet (Fig. S22). Nonetheless, essential regions of the enzymes as active sites seem to be under strong purifying selection, suggesting preservation of the ancestral function. The observation that DmdA sequences have more divergent sites than non-DmdA sequences suggest that non-DmdA conserves the ancestral function, whereas DmdA evolved to acquire new functions in different environments, probably as a response to the Huronia ice ball Earth (Zhang, 2003).

\section{Second scenario: coevolution between Roseobacter and DMSP-producing-phytoplankton}

In the second scenario, our results do not support the hypothesis of a co-evolution scenario between Roseobacter and DMSP-producing-phytoplankton (Luo et al., 2013). On the contrary, we found an ancestor sequence of DmdA cluster similar to DmdA from a strain of Ca. P. ubique that diverged after a more recent duplication event (Fig. 7.A and Fig. S10), before the dinoflagellate radiation in the late Permian (Fig. 4). This finding indicates that the enzyme activity has not changed in the course of DmdA evolution and is in Roseobacter because their genome expansion (250 mya) provided a new trait to use DMSP produced by phytoplankton during its diversification. Indeed, we found that most of the codons in DmdA clade are under purifying selection, probably due to the importance of this pathway for sulfur acquisition. Nonetheless, we also detected episodic positive selection in four sequences affecting a few sites, suggesting that adaptive evolution fine-tuned the function of DmdA in Roseobacter and other types of Alphaproteobacteria (like HIMB59 and Hoeflea). Furthermore, positively selected residues were located around the GcvT domain and close to the residue involved in conserved interaction with THF (Fig. 6), reinforcing the idea of adaptive evolution in response to the external environment. 
582 During the study of this scenario, we suspected that $d m d A$ was acquired by HGT in Roseobacter

583 and SAR11 (Fig. S8). This agrees with Luo et al., (2013) and Tang et al. (2010) which found that

584 the expansion of $d m d A$ resulted from HGT events. According to our phylogeny, the ancestral

$585 d m d A$ sequence originated as a results of HGT (in individuals not connected by inheritance that

586 acquired the $d m d A$ ancestral sequence) from other marine heterotrophic bacteria, that during the

587 Archean adapted to the presence of DMSP. However, after the HGT events, some $d m d A$

588 sequences have acquired similar residue changes by independent (parallel) evolution, reinforcing

589 the idea of functional/ecological constrains. Therefore, Rhodobacteraceae can live in an

590 environment where DMSP is the main source of sulfur because they acquired the $d m d A$ ancestor

591 sequence by HGT, prior to having been exposed to the environment in which the DmdA protein

592 proved useful, as Luo \& Moran (2014) suggested. We did not find any signal of positive selection

593 in the Roseobacter group, but in contrast we found episodic evolution between SAR11 sequences.

594 Yet, as we already mentioned, DMSP is part of an ancient pathway in Alphaproteobacteria

595 (Bullock et al., 2017) and it could explain the ancient origin of DmdA.

596

597

On the other hand, Roseobacter orthologs analyzed in this study were functionally annotated as

598

599

600

601

602

603

604

605

606

607

608 However, we identified orthologs within DmdA gene family as Sánchez-Pérez et al (2008) proposed in their study regarding related genes that perform the same cellular function, but apparently under different ecological conditions, as we found differences in predicted isoelectric point values (pI) (Table S7). Nandi et al. (2005) results also support that orthologs with very variable pI values may be taken as markers to predict the organism's ecological niche. We suggest the name "eco-orthologs", similar to the ecoparalogs describe by Sánchez-Pérez et al (2008) in their study of the halophilic species Salinibacter ruber. The pI values of a protein provide an indication of its acidic nature on the surface, corresponding to its optimal activity and stability at high salinity (Oren et al., 2005; Sánchez-Pérez et al, 2008). Therefore, proteins that differ in their acid residue content on their surface, and consequently in their predicted pI values and halophilicities are considered eco-orthologs (Nandi et al., 2005; Oren et al., 2005; SánchezPérez et al, 2008). We observed the highest pI values in the DmdA ancestor sequences, as well as in Ca. P. ubique DmdA (Fig. 7.A; red color). Therefore, we deduce that the DmdA ancestor was adapted to a higher salinity, which could have modulated the selection of the DMSP enzymatic degradation routes as in bacteria such as the model organism $R$. pomeroyi DSS-3 (Salgado et al., 2014). Interestingly, $R$. pomeroyi degrades more DMSP by the demethylation pathway under high salinity conditions, releasing a higher amount of MeSH (Howard et al., 2008; Magalhães et al., 2012; Salgado et al., 2014). The success of the $d m d A$ gene could be explained if we consider that the environment evolved from higher to lower salinity conditions. Under this environment, $d m d A$ would have been kept without important changes in its structure, sequence, function and $\mathrm{K}_{\mathrm{m}}$ value and even would be essential for the degradation of the large amounts of DMSP produced by phytoplankton. Indeed, it would be interesting to evaluate $\mathrm{K}_{\mathrm{m}}$ values among ancestral proteins of DmdA and their descendants to support the key role of $K_{m}$ during DmdA evolution. In addition, since $d m d A$ seems to be part of a conserved operon (González et al., 2019), its evolution might be linked to genes such as $d m d B, d m d C$ and $d m d D$ that encode part of the enzymes for the rest of the pathway.

Given our data, we propose that the ancestor of the pathway that evolved during the Archean was exposed to a higher concentration of DMSP in a sulfur-rich atmosphere and in an anoxic ocean, compared to recent eco-ortologs which should adapt to lower concentration of DMSP (Fig 7.A: blue color). Indeed, the ancestral eco-orthologs from which recent eco-orthologs derived (Candidatus Puniceispirilum marinum IMCC1322, ADE38317.1 and the Roseobacter clade) 
631

632

633

634

635

636

637

638

639

640

641

642

643

644

645

646

647

648

649

650

651

652

653

654

655

656

657

658

659

660

661

662

663

664

665

666

667

668

669

670

671

672

673

674

675

676

could have undergone episodes of adaptation (the branch showed positive selection in branchmodels) which would explain the change in protein stability (Pál et al., 2006). As consequence, the protein could have experienced slight reductions or loss of function.

\section{Third scenario: pre-adapted enzymes to DMSP prior to Roseobacter origin}

In this evolutionary scenario, the Roseobacter clade was pre-adapted to the conditions created by eukaryotic phytoplankton of the late Permian, including dinoflagellates that released vast amounts of DMSP (Bullock et al., 2017; Luo \& Moran, 2014). Our analyses indicate that the Roseobacter ancestor was already adapted to a high DMSP before the Roseobacter clade arose (Luo et al., 2013). Therefore, we support Reisch et al. (2011 a,b) hypothesis that DMSP demethylation pathway enzymes are an adapted versions of enzymes that were already in bacterial genomes and that evolved in response to the availability of DMSP. Since the first step in DMSP demethylation is a reaction catalyzed by DMSP demethylase encoded by $d m d A$ gene (Dickschat et al., 2015), DMSP adaptation could have been evolved in this gene that originated in the Archean, a time where several lineages of bacteria produced DMSP as an osmolyte or antioxidant in the presence of the early cyanobacteria, or as a cryoprotectant in the Huronian glaciation. In bacteria, a methyltransferase gene, $d y s B$, is up-regulated during increased salinity, nitrogen limitation, and at low temperatures (Curson et al., 2017), conditions already predicted to stimulate DMSP production in phytoplankton and algae (Bullock, et al., 2017; Ito, et al., 2011). Afterward, those roles may have helped to drive the fine adaptation of existing enzymes for DMSP metabolism, and those adaptations came handy in the late Precambrian glaciations that allowed the radiation of algae and animals.

\section{Conclusions}

In conclusion, we found that Roseobacter adaptation to DMSP occurred via functional diversification after duplication events of the $d m d A$ gene and adaptations to environmental variations via eco-orthologs of intermediate divergence. Our findings suggest that the DmdA ancestor evolved to play a key role in the ocean sulfur cycle due to a shift in salinity concentration, which involved a change in DMSP synthesis.

\section{Acknowledgements}

We would like to thank to Dr. Romain Studer from BenevolentAI for his critical role in the 3D visualization of protein and mapping sites onto the 3D structure and Dr. Buckley Iglesias from Universidad Autonóma de Madrid for his introduction to molecular dating analysis with BEAST 2. This research was supported by grant CTM2016-80095-C2 from the Spanish Ministry of Economy and Competitiveness.

\section{References}

Anisimova, M., Bielawski, J. P., \& Yang, Z. (2001). Accuracy and power of the Likelihood Ratio Test in Detecting Adaptive Molecular Evolution. Molecular Biology and Evolution, 18(8), 15851592. https://doi.org/10.1093/oxfordjournals.molbev.a003945 
677

678

679

680

681

682

683

684

685

686

687

688

689

690

691

692

693

694

695

696

697

698

699

700

701

702

703

704

705

706

707

708

709

710

711

712

713

714

715

716

717

718

719

720

721

722

723

724

725
Ashkenazy, H., Penn, O., Doron-Faigenboim, A., Cohen, O., Cannarozzi, G., Zomer, O., \& Pupko, T. (2012). FastML: a web server for probabilistic reconstruction of ancestral sequences. Nucleic Acids Research, 40(W1), W580-W584. https://doi.org/10.1093/nar/gks498

Berman, H. M., Westbrook, J., Feng, Z., Gilliland, G., Bhat, T. N., Weissig, H., Shindyalov, I. N. \& Bourne, P. E. (2000). The protein Data Bank. Nucleic Acids Research, 28(1), 235-242. https:// doi.org/10.1093/nar/28.1.235

Bielawski, J. P., \& Yang, Z. (2004). A maximum Likelihood Method for Detecting Functional Divergence at Individual Codon Sites, with Application to Gene Family Evolution. Journal of Molecular Evolution, 59(1). https://doi.org/10.1007/s00239-004-2597-8

Bouckaert, R., Heled, J., Kühnert, D., Vaughan, T., Wu, C.-H., Xie, D., Suchard, M. A., Rambaut, A., Drummond, A. J. (2014). BEAST 2: A software Platform for Bayesian Evolutionary Analysis. PLoS Computational Biology, 10(4), e1003537. https://doi.org/10.1371/journal.pcbi.1003537

Bullock, H. A., Luo, H., \& Whitman, W. B. (2017). Evolution of dimethylsulfoniopropionate Metabolism in Marine Phytoplankton and Bacteria. Frontiers in Microbiology, 8. https://doi.org/ 10.3389/fmicb.2017.00637

Bürgmann, H., Howard, E. C., Ye, W., Sun, F., Sun, S., Napierala, S., \& Moran, M. A. (2007). Transcriptional response of Silicibacter pomeroyi DSS-3 to dimethylsulfoniopropionate (DMSP). Environmental Microbiology, 9(11), 2742-2755. https://doi.org/10.1111/j.14622920.2007.01386.x

Capella-Gutiérrez, S., Silla-Martínez, J. M., \& Gabaldon, T. (2009). trimAl: a tool for automated alignment trimming in large-scale phylogenetic analyses. Bioinformatics, 25(15), 1972-1973. https://doi.org/10.1093/bioinformatics/btp348

Castresana, J. (2000). Selection of conserved Blocks from Multiple Alignments for Their Use in Phylogenetic Analysis. Molecular Biology and Evolution, 17(4), 540-552.

https://doi.org/10.1093/oxfordjournals.molbev.a026334

Clamp, M., Cuff, J., Searle, S.M., Barton, G.J. (2004). The Jalview Java alignment editor.

Bioinformatics, 20(3), 426-427. https://doi.org/10.1093/bioinformatics/btg430

Curson, A. R. J., Todd, J. D., Sullivan, M. J., \& Johnston, A. W. B. (2011). Catabolism of dimethylsulphoniopropionate: microorganisms, enzymes and genes. Nature Reviews Microbiology, 9, 849. https://doi.org/10.1038/nrmicro2653

Curson, A. R. J., Liu, J., Martínez, A. B., Green, R. T., Chan, Y., Carrión, O., Williams, B. T. Zhang, S-H., Yang, G-P., Page, P. C. B., Zhang, X-H \& Todd, J. D. (2017).

Dimethylsulfoniopropionate biosynthesis in marine bacteria and identification of the key gene in this process. Nature Microbiology, 2(17009). https://doi.org/10.1038/nmicrobiol2017.9

Darriba, D., Taboada, G. L., Doallo, R., \& Posada, D. (2011). ProtTest 3: fast selection of best-fit models of protein evolution. Bioinformatics, 27(8), 1164-1165.

https://doi.org/10.1093/bioinformatics/btr088 
726

727

728

729

730

731

732

733

734

735

736

737

738

739

740

741

742

743

744

745

746

747

748

749

750

751

752

753

754

755

756

757

758

759

760

761

762

763

764

765

766

767

768

769

770

771

772

773
Darriba, D., Taboada, G. L., Doallo, R., \& Posada, D. (2012). jModelTest 2: more models, new heuristics and parallel computing. Nature Methods, 9(8), 772-772.

https://doi.org/10.1038/nmeth.2109

DeLano, W. L. (2002). Pymol: An open-source molecular graphics tool. CCP4 Newsletter On Protein Crystallography, 40(1), 82-92

Dickschat, J. S., Rabe, P., \& Citron, C. A. (2015). The chemical biology of dimethylsulfoniopropionate. Organic \& Biomolecular Chemistry, 13(7), 1954-1968. https://doi.org/10.1039/C4OB02407A

Edgar, R. C. (2004). MUSCLE: multiple sequence alignment with high accuracy and high throughput. Nucleic Acids Research, 32(5), 1792-1797. https://doi.org/10.1093/nar/gkh340

Felsenstein, J. (1981). Evolutionary trees from DNA sequences: A maximum likelihood approach. Journal of Molecular Evolution, 17(6), 368-376. https://doi.org/10.1007/BF01734359

Finn, R. D., Mistry, J., Tate, J., Coggill, P., Heger, A., Pollington, J. E., Gavin, O. L., Gunasekaran, P., Ceric, G., Forslund, K., Holm, L., Sonnhammer, E. L. L., Eddy, S. R. \& Bateman, A. (2010). The Pfam protein families database. Nucleic Acids Research, 38(suppl_1), D211-D222. https://doi.org/10.1093/nar/gkp985

Galinski, E. A. (1995). Osmoadaptation in bacteria. In Advances in Microbial Physiology (Vol. 37, pp. 273-328). https://doi.org/10.1016/S0065-2911(08)60148-4

Gasteiger, E., Hoogland, C., Gattiker, A., Duvaud, S., Wilkins, M. R., Appel, R. D., \& Bairoch, A. (2005). Protein identification and Analysis Tools on the ExPASy Server. In J. M. Walker (Ed.), The Proteomics Protocols Handbook (pp. 571-607). https://doi.org/10.1385/1-59259-890$0: 571$

Gernhard, T. (2008). The conditioned reconstructed process. Journal of Theoretical Biology, 253(4), 769-778. https://doi.org/10.1016/j.jtbi.2008.04.005

González, J. M. G., Kiene, R. P., \& Moran, M. A. (1999). Transformation of sulfur Compounds by an Abundant Lineage of Marine Bacteria in the Subclass of the Class Proteobacteria. Appl. Environ. Microbiol., 65, 10.

González, J. M. (2003). Silicibacter pomeroyi sp. nov. and Roseovarius nubinhibens sp. nov., dimethylsulfoniopropionate-demethylating bacteria from marine environments. International Journal of Systematic and Evolutionary Microbiology, 53(5), 1261-1269. https://doi.org/10.1099/ijs.0.02491-0

González, J. M., Hernández, L., Manzano, I., \& Pedrós-Alió, C. (2019). Functional annotation of orthologs in metagenomes: a case study of genes for the transformation of oceanic dimethylsulfoniopropionate. The ISME Journal, 13(5), 1183-1197.

https://doi.org/10.1038/s41396-019-0347-6 
774 Guindon, S., Dufayard, J.-F., Lefort, V., Anisimova, M., Hordijk, W., \& Gascuel, O. (2010).

775 New algorithms and Methods to Estimate Maximum-Likelihood Phylogenies: Assessing the

776 Performance of PhyML 3.0. Systematic Biology, 59(3), 307-321.

777 https://doi.org/10.1093/sysbio/syq010

778

779

780

781

782

783

784

785

786

787

788

789

790

791

792

793

794

795

796

797

798

799

800

801

802

803

804

805

806

807

808

809

810

811

812

813

814

815

816

817

818

819

820

821
Hedges, S. B., Dudley, J., \& Kumar, S. (2006). TimeTree: a public knowledge-base of divergence times among organisms. Bioinformatics, 22(23), 2971-2972. https://doi.org/10.1093/ bioinformatics/btl505

Hedges, S. Blair, Marin, J., Suleski, M., Paymer, M., \& Kumar, S. (2015). Tree of life Reveals Clock-Like Speciation and Diversification. Molecular Biology and Evolution, 32(4), 835-845. https://doi.org/10.1093/molbev/msv037

Howard, E. C., Henriksen, J. R., Buchan, A., Reisch, C. R., Burgmann, H., Welsh, R., Wenying, Y., González, J. M., Mace, K., Joye, S. B., Kiene, R. P., Whitman, W. B. \& Moran, M. A. (2006). Bacterial taxa That Limit Sulfur Flux from the Ocean. Science, 314(5799), 649-652. https://doi.org/10.1126/science.1130657

Howard, E. C., Sun, S., Biers, E. J., \& Moran, M. A. (2008). Abundant and diverse bacteria involved in DMSP degradation in marine surface waters. Environmental Microbiology, 10(9), 2397-2410. https://doi.org/10.1111/j.1462-2920.2008.01665.x

Huelsenbeck, J. P., \& Rannala, B. (1997). Phylogenetic methods Come of Age: Testing Hypotheses in an Evolutionary Context. Science, 276(5310), 227.

https://doi.org/10.1126/science.276.5310.227

Hug, L. A., Baker, B. J., Anantharaman, K., Brown, C. T., Probst, A. J., Castelle, C. J., Butterfield, C. N., Hernsdorf, A. W., Amano, Y., Ise, K., Suzuki, Y., Dudek, N., Relman, D. A., Finstad, K. M., Amundson, R., Thomas, B. C. \& Banfield, J. F. (2016). A new view of the tree of life. Nature Microbiology, 1, 16048. https://doi.org/10.1038/nmicrobiol.2016.48

Ito, T., Asano, Y., Tanaka, Y., Takabe, T. (2011). Regulation of biosynthesis of dimethylsulfoniopropionate and its uptake in sterile mutant of Ulva pertusa (Chlorophyta). Journal of Phycology, 47(3), 517-523. https://doi.org/10.1111/j.1529-8817.2011.00977.x

Kiene, R. P., Linn, L. J., González, J. G., Moran, M. A., \& Bruton, J. A. (1999).

Dimethylsulfoniopropionate and methanethiol Are Important Precursors of Methionine and Protein-Sulfur in Marine Bacterioplankton. Applied and Environmental Microbiology, 65(10), 4549-4558.

Kinoshita, K., \& Nakamura, H. (2003). Protein informatics towards function identification. Current Opinion in Structural Biology, 13, 296-400. https://doi.org/10.1016/s0959440x(03)00074-5

Kishino, H., \& Hasegawa, M. (1989). Evaluation of the maximum likelihood estimate of the evolutionary tree topologies from DNA sequence data, and the branching order in hominoidea. Journal of Molecular Evolution, 29(2), 170-179. https://doi.org/10.1007/BF0210011 
822 Klemetsen, T., Raknes, I. A., Fu, J., Agafonov, A., Balasundaram, S. V., Tartari, G., Robertsen,

823 E. \& Willassen, N. P. (2018). The MAR databases: development and implementation of

824 databases specific for marine metagenomics. Nucleic Acids Research, 46(D1), D692-D699.

825 https://doi.org/10.1093/nar/gkx1036

826

827

Kumar, S., Stecher, G., \& Tamura, K. (2016). MEGA7: Molecular evolutionary Genetics

828

829

830

831

832

833

834

835

836

837

838

839

840

841

842

843

844

845

846

847

848

849

850

851

852

853

854

855

856

857

858

859

860

861

862

863

864

865

866

867

868

869

Analysis Version 7.0 for Bigger Datasets. Molecular Biology and Evolution, 33(7), 1870-1874.

https://doi.org/10.1093/molbev/msw054

Kumar, S., Stecher, G., Suleski, M., \& Hedges, S. B. (2017). TimeTree: A resource for

Timelines, Timetrees, and Divergence Times. Molecular Biology and Evolution, 34(7), 1812-

1819. https://doi.org/10.1093/molbev/msx116

Lanfear, R., Frandsen, P. B., Wright, A. M., Senfeld, T., \& Calcott, B. (2016). PartitionFinder 2: New methods for Selecting Partitioned Models of Evolution for Molecular and Morphological Phylogenetic Analyses. Molecular Biology and Evolution, msw260.

https://doi.org/10.1093/molbev/msw260

Lartillot, N., \& Philippe, H. (2004). A Bayesian mixture Model for Across-Site Heterogeneities in the Amino-Acid Replacement Process. Molecular Biology and Evolution, 21(6), 1095-1109. https://doi.org/10.1093/molbev/msh112

Lartillot, N., \& Philippe, H. (2006). Computing Bayes factors Using Thermodynamic Integration. Systematic Biology, 55(2), 195-207. https://doi.org/10.1080/10635150500433722

Lartillot, N., Brinkmann, H., \& Philippe, H. (2007). Suppression of long-branch attraction artefacts in the animal phylogeny using a site-heterogeneous model. BMC Evolutionary Biology, 7(Suppl 1), S4. https://doi.org/10.1186/1471-2148-7-S1-S4

Le, S. Q., \& Gascuel, O. (2008). An improved General Amino Acid Replacement Matrix. Molecular Biology and Evolution, 25(7), 1307-1320. https://doi.org/10.1093/molbev/msn067

Lovelock, J. E. (1983). Gaia as seen Through the Atmosphere. In P. Westbroek \& E. W. de Jong (Eds.), Biomineralization and Biological Metal Accumulation (pp. 15-25).

https://doi.org/10.1007/978-94-009-7944-4_2

Luo, H., Csüros, M., Hughes, A. L., \& Moran, M. A. (2013). Evolution of Divergent Life History Strategies in Marine Alphaproteobacteria. MBio, 4(4). https://doi.org/10.1128/mBio.00373-13

Luo, H., \& Moran, M. A. (2014). Evolutionary ecology of the Marine Roseobacter Clade. Microbiology and Molecular Biology Reviews, 78(4), 573-587.

https://doi.org/10.1128/MMBR.00020-14

Magalhães, C., Salgado, P., Kiene, R. P., \& Bordalo, A. A. (2012). Influence of salinity on dimethyl sulfide and methanethiol formation in estuarine sediments and its side effect on nitrous oxide emissions. Biogeochemistry, 110(1-3), 75-86. https://doi.org/10.1007/s10533-011-9690-z

Moran, M. A., Belas, R., Schell, M. A., González, J. M., Sun, F., Sun, S., Binder, B. J., Edmonds, 870 
872

873

874

875

876

877

878

879

880

881

882

883

884

885

886

887

888

889

890

891

892

893

894

895

896

897

898

899

900

901

902

903

904

905

906

907

908

909

910

911

912

913

914

915

916

917

918

919
I., Ulrich, L. E., Thompson, L. S., Saunders, E., \& Buchan, A. (2007). Ecological genomics of Marine Roseobacters. Applied and Environmental Microbiology, 73(14), 4559-4569. https://doi.org/10.1128/AEM.02580-06

Nandi, S., Mehra, N., Lynn, A., Bhattacharya, A. (2005). Comparison of theoretical proteome: Identification of COGs with conserved and variable PI with multimodal PI distribution. BMC Genomics, 6(116). https://doi.org/10.1186/1471-2164-6-116

Nelson, A. D. L., Haug-Baltzell, A. K., Davey, S., Gregory, B. D., \& Lyons, E. (2018). EPICCoGe: managing and analyzing genomic data. Bioinformatics, 34(15), 2651-2653. https://doi.org/10.1093/bioinformatics/bty106

Newton, R. J., Griffin, L. E., Bowles, K. M., Meile, C., Gifford, S., Givens, C. E., Howard, E. C., King, E., Oakley, C. A., Reisch, C. R., Rinta-Kanto, J. M., Sharma, S., Sun, S., Varaljay, V., Vila-Costa, M., Westrich, J. R. \& Moran, M. A. (2010). Genome characteristics of a generalist marine bacterial lineage. The ISME Journal, 4(6), 784-798.

https://doi.org/10.1038/ismej.2009.150

Nguyen, L. T., Schmidt, H. A., von Haeseler, A., \& Minh, B. Q. (2015). IQ-TREE: A fast and Effective Stochastic Algorithm for Estimating Maximum-Likelihood Phylogenies. Molecular Biology and Evolution, 32(1), 268-274. https://doi.org/10.1093/molbev/msu300

Oren, A., Larimer, F., Richardson, P., Lapidus, A., \& Csonka, L. N. (2005). How to be moderately halophilic with broad salt tolerance: clues from the genome of Chromohalobacter salexigens. Extremophiles, 9(4), 275-279. https://doi.org/10.1007/s00792-005-0442-7

Pál, C., Papp, B., \& Lercher, M. J. (2006). An integrated view of protein evolution. Nature Reviews Genetics, 7(5), 337-348. https://doi.org/10.1038/nrg1838

Puigbo, P., García-Vallve, S., \& McInerney, J. O. (2007). TOPD/FMTS: a new software to compare phylogenetic trees. Bioinformatics, 23(12), 1556-1558.

https://doi.org/10.1093/bioinformatics/btm135

R Core Team. (2017). R: A language and environment for statistical computing. $\mathrm{R}$ foundation for Statistical Computing, Vienna, Austria. URL https://www.R-project.org/

Raina, J.-B., Dinsdale, E. A., Willis, B. L., \& Bourne, D. G. (2010). Do the organic sulfur compounds DMSP and DMS drive coral microbial associations? Trends in Microbiology, 18(3), 101-108. https://doi.org/10.1016/j.tim.2009.12.002

Rambaut, A., \& Drummond, A.J. (2002, 2010). TreeAnnotator, v1.6.1. Available from http://beast.bio.ed.ac.uk/.

Rambaut, A. (2009). FigTree, version 1.4.3. Available from

http://tree.bio.ed.ac.uk/software/figtree.

Rambaut, A., \& Drummond, A.J. (2013). Tracer v1.6. Available from: URL http://beast.bio.ed.ac.uk/Tracer. 
920 Ravenhall, M., Škunca, N., Lassalle, F., \& Dessimoz, C. (2015). Inferring horizontal Gene

921 Transfer. PLOS Computational Biology, 11(5), e1004095.

922 https://doi.org/10.1371/journal.pcbi.1004095

923

924

925

926

927

928

929

930

931

932

933

934

935

936

937

938

939

940

941

942

943

944

945

946

947

948

949

950

951

952

953

954

955

956

957

958

959

960

961

962

963

964

965

966

967

968
Reisch, C. R., Moran, M. A., \& Whitman, W. B. (2008). Dimethylsulfoniopropionate-dependent Demethylase (DmdA) from Pelagibacter ubique and Silicibacter pomeroyi. Journal of Bacteriology, 190(24), 8018-8024. https://doi.org/10.1128/JB.00770-08

Reisch, C. R., Moran, M. A., \& Whitman, W. B. (2011a). Bacterial catabolism of Dimethylsulfoniopropionate (DMSP). Frontiers in Microbiology, 2. https://doi.org/10.3389/fmicb.2011.00172

Reisch, C. R., Stoudemayer, M.J., Varaljay, V.A., Amster, I.J., Moran, M.A., \& Whitman, W.B. (2011b). Novel pathway for assimilation of dimethylsulphoniopropionate widespread in marine bacteria. Nature, 473, 208-211. https://doi.org/10/1038/nature10078

Rost, B. (2002). Enzyme function less conserved than anticipated. Journal of Molecular Biology, 318(2), 595-608. https://doi.org/10.1016/s0022-2836(02)0016-5

Roy, A., Kucukural, A., \& Zhang, Y. (2010). I-TASSER: a unified platform for automated protein structure and function prediction. Nature Protocols, 5(4), 725-738.

https://doi.org/10.1038/nprot.2010.5

Salgado, P., Kiene, R., Wiebe, W., \& Magalhães, C. (2014). Salinity as a regulator of DMSP degradation in Ruegeria pomeroyi DSS-3. Journal of Microbiology, 52(11), 948-954.

https://doi.org/10.1007/s12275-014-4409-1

Sánchez-Pérez, G., Mira, A., Nyirö, G., Pasić, L., \& Rodríguez-Valera, F. (2008). Adapting to environmental changes using specialized paralogs. Trends in Genetics, 24(4), 154-158.

https://doi.org/10.1016/j.tig.2008.01.002

Schliep, K.P. (2011). phangorn: phylogenetic analysis in R. Bioinformatics, 27(4), 592-593. https://doi.org/10.1093/bioinformatics/btg706

Schuller, D. J., Reisch, C. R., Moran, M. A., Whitman, W. B., \& Lanzilotta, W. N. (2012). Structures of dimethylsulfoniopropionate-dependent demethylase from the marine organism Pelagabacter ubique: Structures and mechanism of DmdA from Pelagabacter ubique. Protein Science, 21(2), 289-298. https://doi.org/10.1002/pro.2015

Shimodaira, H., \& Hasegawa, M. (1999). Multiple comparisons of Log-Likelihoods with Applications to Phylogenetic Inference. Molecular Biology and Evolution, 16(8), 1114-1116. https://doi.org/10.1093/oxfordjournals.molbev.a026201

Shimodaira, H., (2002). An approximately Unbiased Test of Phylogenetic Tree Selection. Systematic Biology, 51(3), 492-508. https://doi.org/10.1080/10635150290069913

Siltberg-Liberies, J., Grahnen, J.A., \& Liberies, D.A. (2011). The evolution of protein structures and structural ensembles under functional constraint. Genes (Basel), 2(4), 748-762.

https://doi.org/10.3390/genes2040748 
969

970

971

972

973

974

975

976

977

978

979

980

981

982

983

984

985

986

987

988

989

990

991

992

993

994

995

996

997

998

999

1000

1001

1002

1003

1004

1005

1006

1007

1008

1009

1010

1011

1012

1013

1014

1015

1016

1017
Stamatakis, A. (2006). Phylogenetic models of rate heterogeneity: a high performance computing perspective. Proceedings of the $20^{\text {th }}$ IEE International Parallel and Distributed Processing Symposium, 253. https://doi.org/10.1109/IPDPS.2006.1639535.

Sun, J., Steindler, L., Thrash, J.C., Halsey, K.H., Smith, D.P., Carter, A.E., Landry, Z.C., Giovannoni, S.J. (2011). One carbon metabolism in SAR11 Pelagic Marine bacteria. PloS One, 6(8):e23973. https://doi.org/10.1371/journal.pone.0023973.

Tang, K., Huang, H., Jiao, N. \& Wu, C. H. (2010). Phylogenomic analysis of Marine Roseobacters. PLoS One, 5(7): e11604. https://doi.org/10.1371/journal.pone.0011604.

Tang, H., Thomas, P., \& Xia, H. (n.d.). Reconstruction of the evolutionary history of gene gains and losses since the last universal common ancestor. ArXiv:1802.06035.

Tripp, H. J., Kitner, J. B., Schwalbach, M. S., Dacey, J. W. H., Wilhelm, L. J., \& Giovannoni, S. J. (2008). SAR11 marine bacteria require exogenous reduced sulphur for growth. Nature, 452, 741. https://doi.org/10.1038/nature06776

Williams B. T., Cowles, K., Martínez, A. B., Curson, A. R. J., Zheng, Y., Liu, J., Newton-Payne, S., Hind, A. J., Li, C-Y., Rivera, P. P. L., Carrión, O., Liu, J., Spurgin, L. G., Brearley, C. A., Mackenzie, B. W., Pinchbeck, B. J., Peng, M., Pratscher, J., Zhang, X-H., Zhang, Y-Z., Murrel, J. C. \& Todd, J. D. (2019). Bacteria are important dimethylsulfoniopropionate producers in coastal sediments. Nature Microbiology, 4, 1815. https://doi.org/10.1038/s41564-019-0527-1

Wu, S., \& Zhang, Y. (2007). LOMETS: A local meta-threading-server for protein structure prediction. Nucleic Acids Research, 35(10), 3375-3382. https://doi.org/10.1093/nar/gkm251

Xia, X. (2001). DAMBE: Software package for Data Analysis in Molecular Biology and Evolution. Journal of Heredity, 92(4), 371-373. https://doi.org/10.1093/jhered/92.4.371

Yang, Z. (1998). Likelihood ratio tests for detecting positive selection and application to primate lysozyme evolution. Molecular Biology and Evolution, 15(5), 568-573.

https://doi.org/10.1093/oxfordjournals.molbev.a025957

Yang, Z. (2005). Bayes empirical Bayes Inference of Amino Acid Sites Under Positive Selection. Molecular Biology and Evolution, 22(4), 1107-1118. https://doi.org/10.1093/molbev/msi097

Yang, Z. (2007). PAML 4: Phylogenetic analysis by Maximum Likelihood. Molecular Biology and Evolution, 24(8), 1586-1591. https://doi.org/10.1093/molbev/msm088

Yang, Z., \& dos Reis, M. (2011). Statistical properties of the Branch-Site Test of Positive Selection. Molecular Biology and Evolution, 28(3), 1217-1228. https://doi.org/10.1093/molbev/ msq303

Yang, J., Yan, R., Roy, A., Xu, D., Poisson, J., \& Zhang, Y. (2015). The I-TASSER suite: protein structure and function prediction. Nature Methods, 12(1), 7-8.

https://doi.org/10.1038/nmeth.3213 
1018 Yoch, D. C. (2002). Dimethylsulfoniopropionate: Its sources, Role in the Marine Food Web, and

1019 Biological Degradation to Dimethylsulfide. Applied and Environmental Microbiology, 68(12),

1020 5804-5815. https://doi.org/10.1128/AEM.68.12.5804-5815.2002

1021

1022

Zakon, H. H. (2002). Convergent evolution on the Molecular Level. Brain, Behavior and

1023

1024

1025

1026

1027

1028

1029

1030

1031

1032

1033

1034

1035

1036

1037

Evolution, 59(5-6), 250-261. https://doi.org/10.1159/000063562

Zhang, J. (2003). Evolution by gene duplication: an update. Trends in Ecology \& Evolution, 18(6), 292-298. https://doi.org/10.1016/S0169-5347(03)00033-8

Zhang, J. (2005). Evaluation of an improved Branch-Site Likelihood Method for Detecting Positive Selection at the Molecular Level. Molecular Biology and Evolution, 22(12), 2472-2479. https://doi.org/10.1093/molbev/msi237

Zhang, Y. (2005). TM-align: a protein structure alignment algorithm based on the TM-score. Nucleic Acids Research, 33(7), 2302-2309. https://doi.org/10.1093/nar/gki524

Zubkov, M. V., Fuchs, B. M., Archer, S. D., Kiene, R. P., Amann, R., \& Burkill, P. H. (2001). Linking the composition of bacterioplankton to rapid turnover of dissolved dimethylsulphoniopropionate in an algal bloom in the North Sea. Environmental Microbiology, 1038 3(5), 304-311. https://doi.org/10.1046/j.1462-2920.2001.00196.x 


\section{Figure 1}

Phylogenetic tree based on 20 DmdA orthologs protein sequences and $184 \mathrm{DmdA}$ homologs using BEAST2 and the same parameters set for molecular dating but with 100 million generations.

DmdA sequences are indicated with green color and closer homologs (the limit to select the closer homologs was set to a maximum e-value of e-80) with brown color. Tip labels include a maximum e-value of e-50.

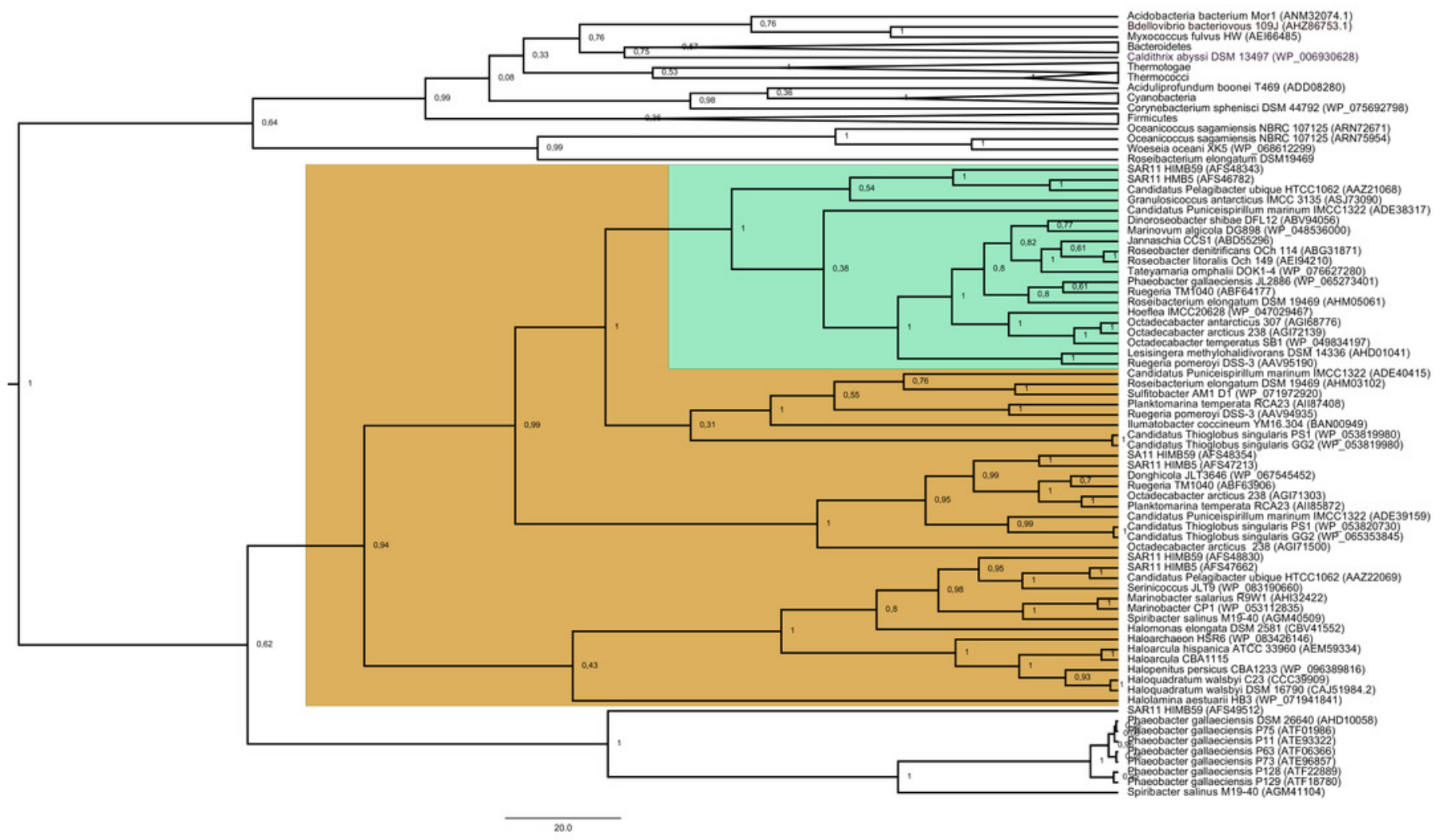




\section{Figure 2}

RAxML phylogenetic tree built with 20 DmdA ortholog protein sequences and $28 \mathrm{DmdA}$ homologs (more information in Table S1).

Non-parametric bootstrap values are shown to establish the support for the clades. DmdA sequences are indicated with blue branches. Tip labels show color according to their taxonomy classification and the asterisk indicates the first gene identified experimentally. Tip labels include a maximum e-value $<$ e-80.

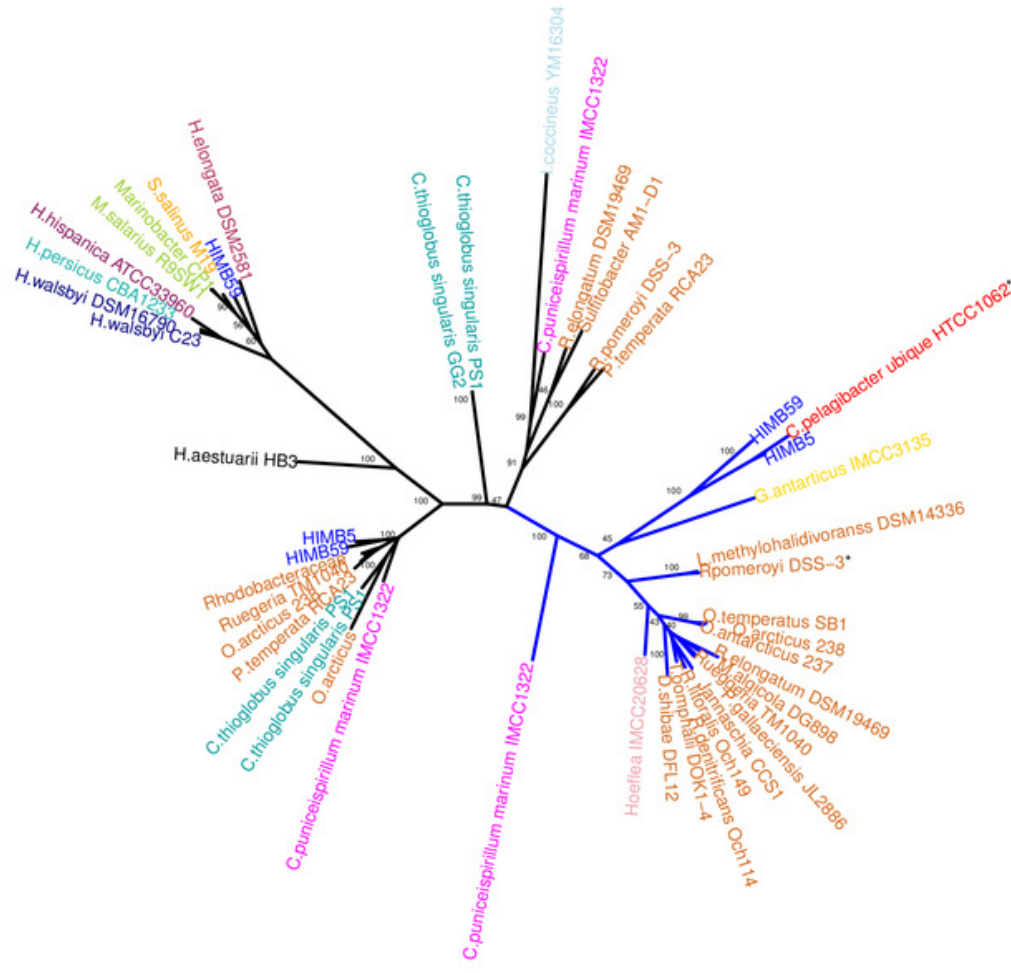

$\Delta$ Alteromonadaceae 
Figure 3

BEAST2 phylogenetic tree constructed with alignment of $20 \mathrm{DmdA}$ ortholog protein sequences and 28 DmdA homologs.

Bayesian posterior probabilities (PP) are shown to establish the support for the clades. Green color indicates DmdA clade. 


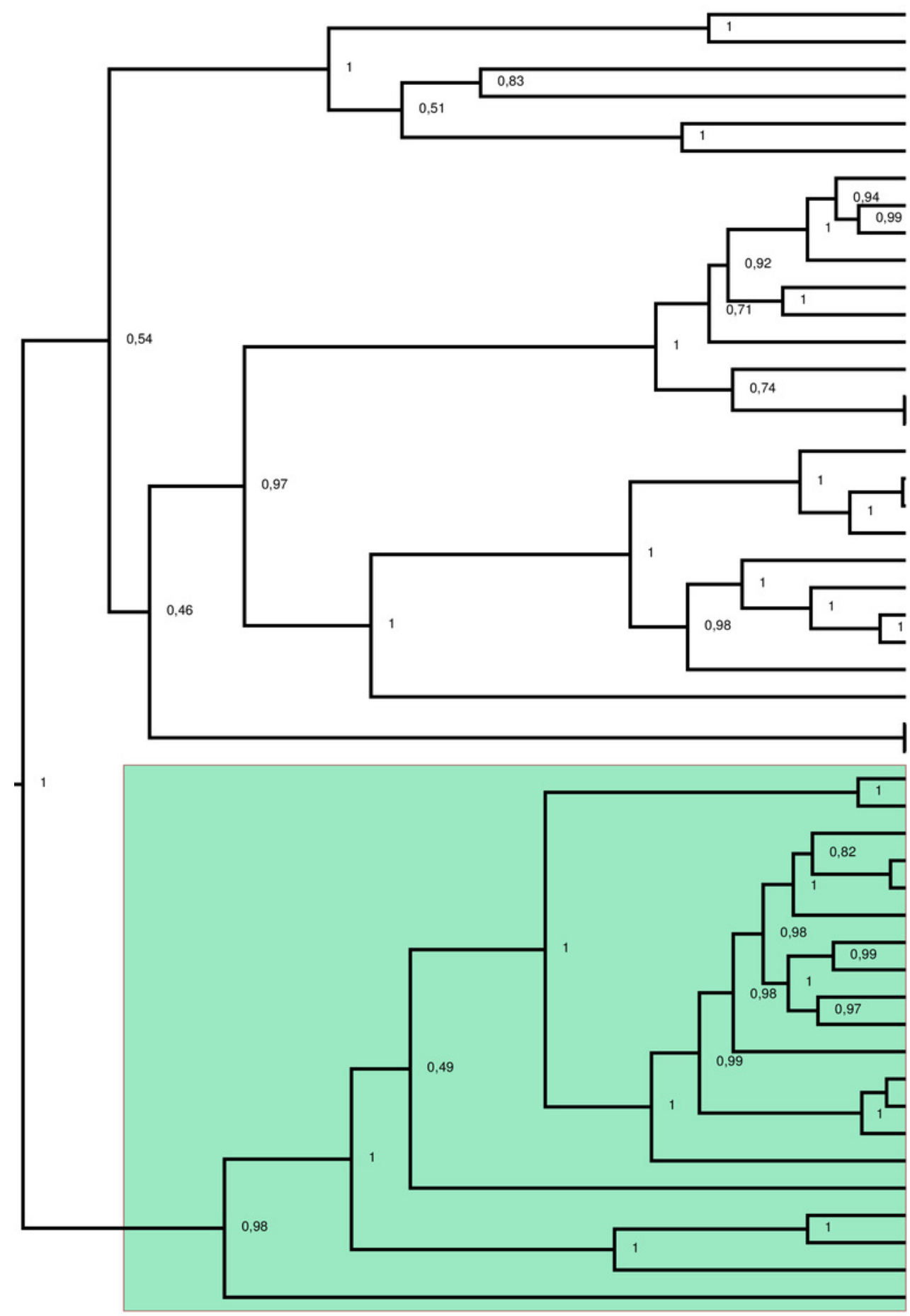

Ruegeria pomeroyi DSS-3

Planktomarina temperata RCA23

Candidatus puniceispirillum marinum IMCC1322

llumatobacter coccineus YM16304

Roseibacterium elongatum DSM19469

Sulfitobacter sp AM1-D1

Ruegeria sp TM1040

Octadecabacter arcticus 238

Planktomarina temperata RCA23

Donghicola JLT3646

HIMB5

HIMB59

Candidatus puniceispirillum marinum IMCC1322

Octadecabacter antarcticus 237

Candidatus thioglobus singularis PS1

Candidatus thioglobus singularis GG2

Haloarcula hispanica ATCC33960

Haloquadratum walsbyi DSM16790

Haloquadratum walsbyi C23

Halopenitus persicus CBA1233

HIMB59

Spiribacter salinus M19-40

Marinobacter salarius R9SW1

Marinobacter sp CP1

Halomonas elongata DSM2581

Halolamina aestuarii HB3

Candidatus thioglobus singularis PS1

Candidatus thioglobus singularis GG2

Ruegeria pomeroyi DSS-3

Leisingera methylohalidivorans DSM14336

Jannaschia sp CCS1

Roseobacter denitrificans Och114

Roseobacter litoralis Och149

Tateyamaria omphalii DOK1-4

Ruegeria sp TM1040

Phaeobacter gallaeciensis JL2886

Roseibacterium elongatum DSM19469

Marinovum algicola DG898

Dinoroseobacter shibae DFL12

Octadecabacter antarcticus 237

Octadecabacter arcticus 238

Octadecabacter temperatus SB1

Hoeflea sp IMCC20628

Granulosicoccus antarcticus IMCC 3135

Candidatus pelagibacter ubique HTCC1062

HIMB5

HIMB59

Candidatus puniceispirillum marinum IMCC1322 


\section{Figure 4}

BEAST2 divergence time estimates from $d m d A$ and non- $d m d A$ genes under uncorrelated relaxed clock model and Birth-death tree model. Absolute time scale in Mya.

Nodes are at mean divergence times and blue bars represent 95\% HPD of node age. Nodes used as calibrated priors in BEAST2 analysis are marked as mrca1, mrca2 and mrca3. Arrows indicate duplication events occurred 1894 Mya (red), 300 Mya (blue), 1000 Mya (green) and 2100 Mya (violet). The predicted non-DmdA clade is shown in brown, DmgdH gene family is in light yellow and the DmdA clade in green color.

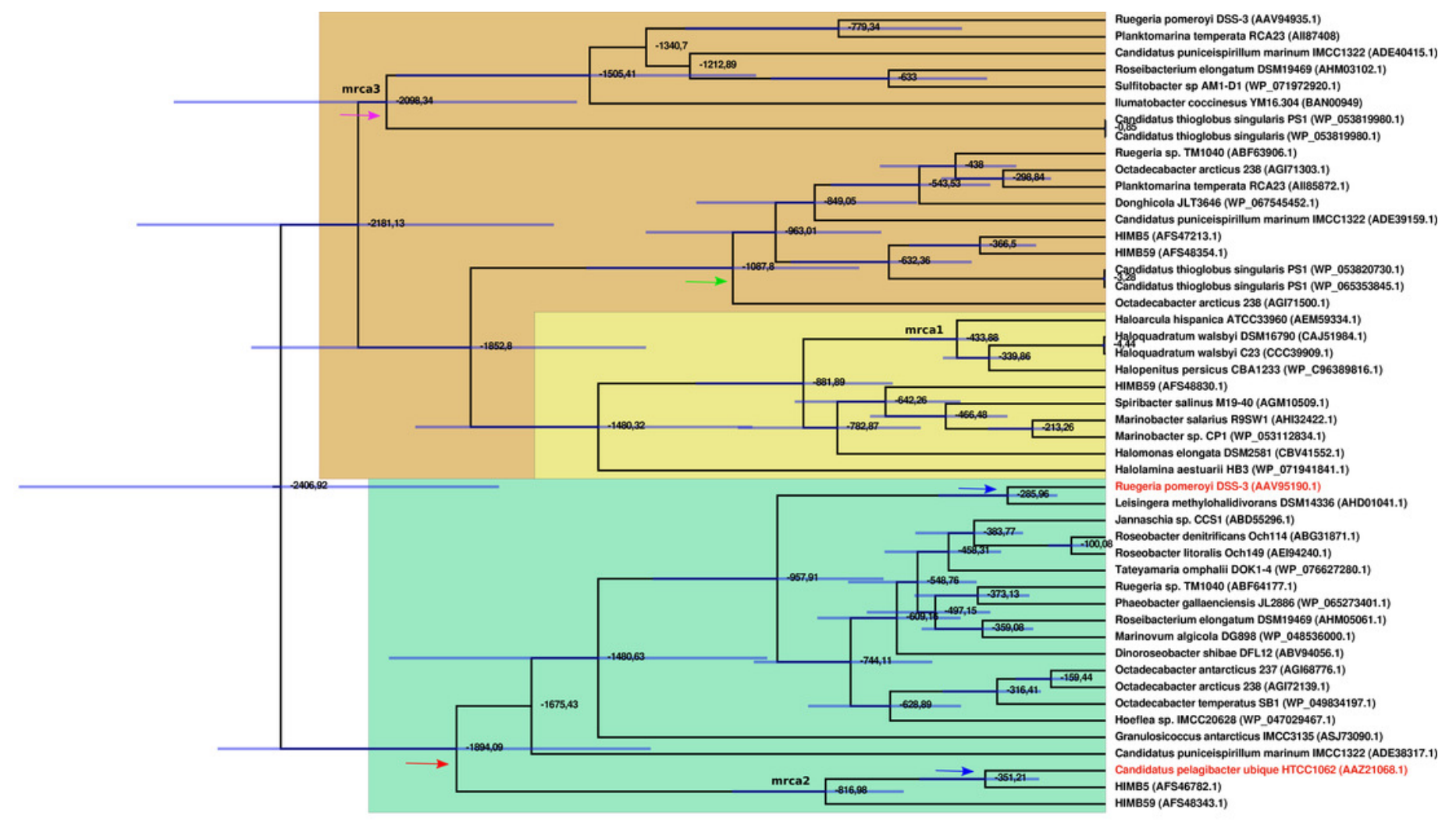




\section{Figure 5}

Posterior probabilities for $\mathrm{dN} / \mathrm{dS}$ categories under the M3 model. Grey, red and blue bars depict the three $\mathrm{dN} / \mathrm{dS}$ categories (values for each category are provide in the key).

Sites that are mostly grey denote codons under strong purifying selection, whereas those predominantly red show codons under weaker purifying selection. Red, blue and grey colors indicate codon sites with $\omega_{2}=0.2483, \omega_{1}=0.06923$ and $\omega_{0}=0.00485$, respectively.

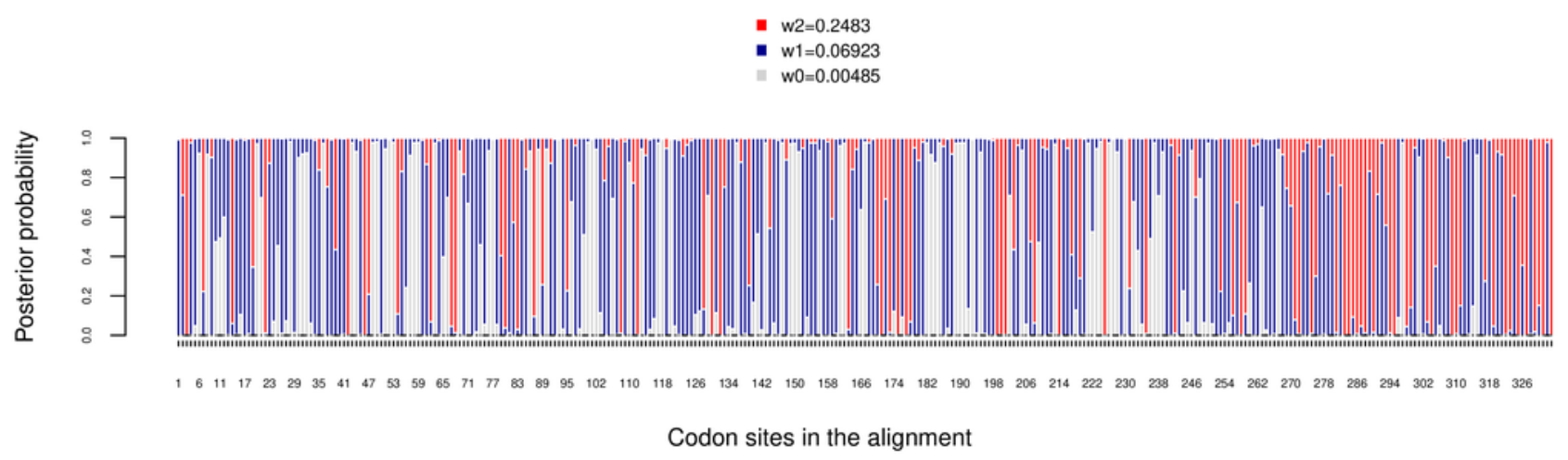


Figure 6

Tertiary structure of DmdA (PBD: 3tfh belongs to Ca. P. ubique) with sites under episodic positive selection.

Sites under positive selection are shown in chain B (white color) as spheres with oxygen in red, nitrogen in blue, hydrogen in white and sulfur in yellow. THF binding sites are shown as yellow sticks.

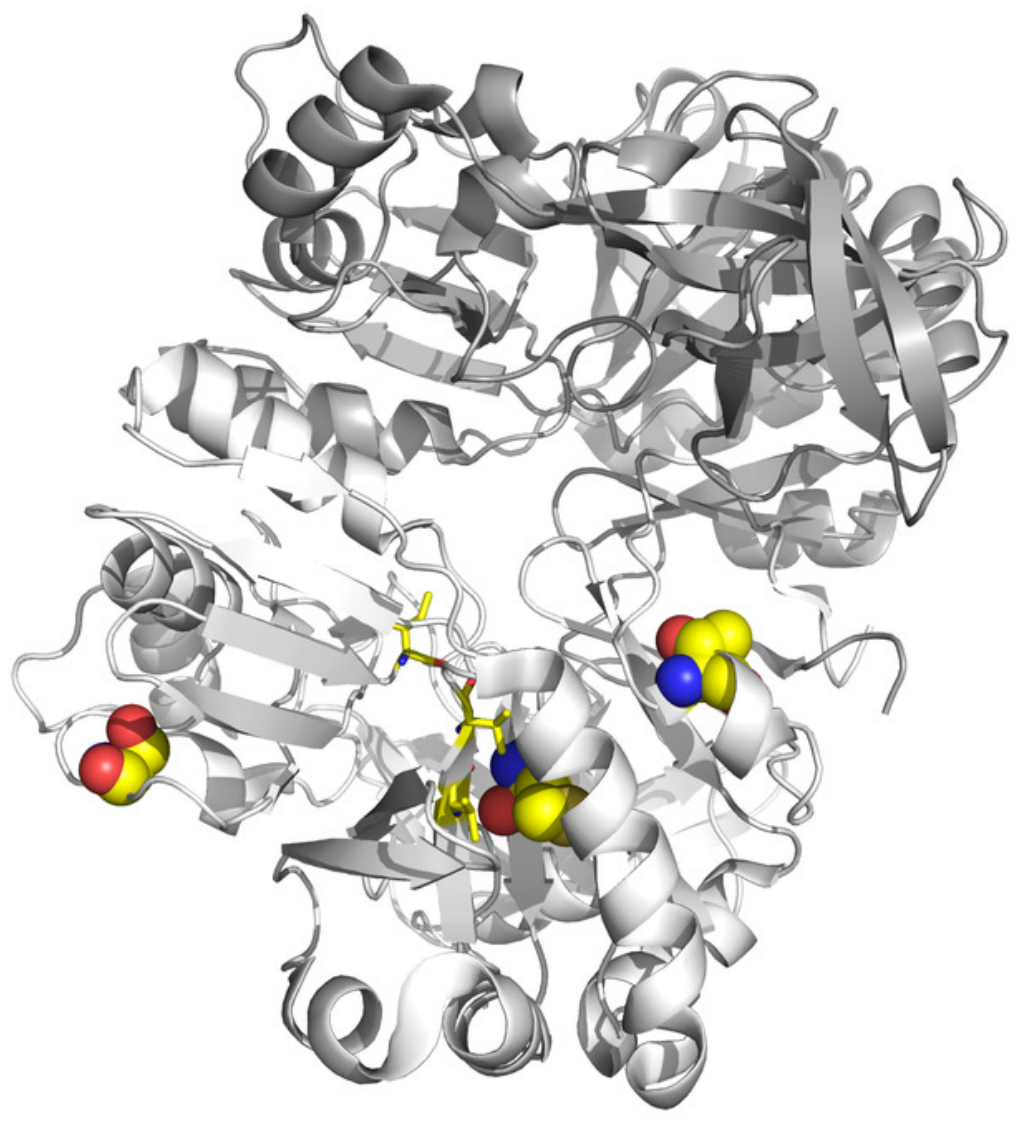




\section{Figure 7}

Hypothesis of DmdA evolution. BI phylogeny under uncorrelated relaxed clock model and Birth-death tree model.

Node names represent the reconstructed ancestral sequences; GcvT family before to main duplication, DmdA for DmdA clade (A) and DmgdH for non-DmdA clade (B). In DmdA clade (A), blue color represents eco-orthologs where pl (predicted isoelectric point value) is $<5.7$ and they are adapted to low concentration of DMSP in comparison with DmdA orthologs (red color) which have $\mathrm{pl}=>6.5$. In non-DmdA clade (B), yellow branches represent homologs with DmgdH tertiary structure and black branches homologs with DmdA tertiary structure. 


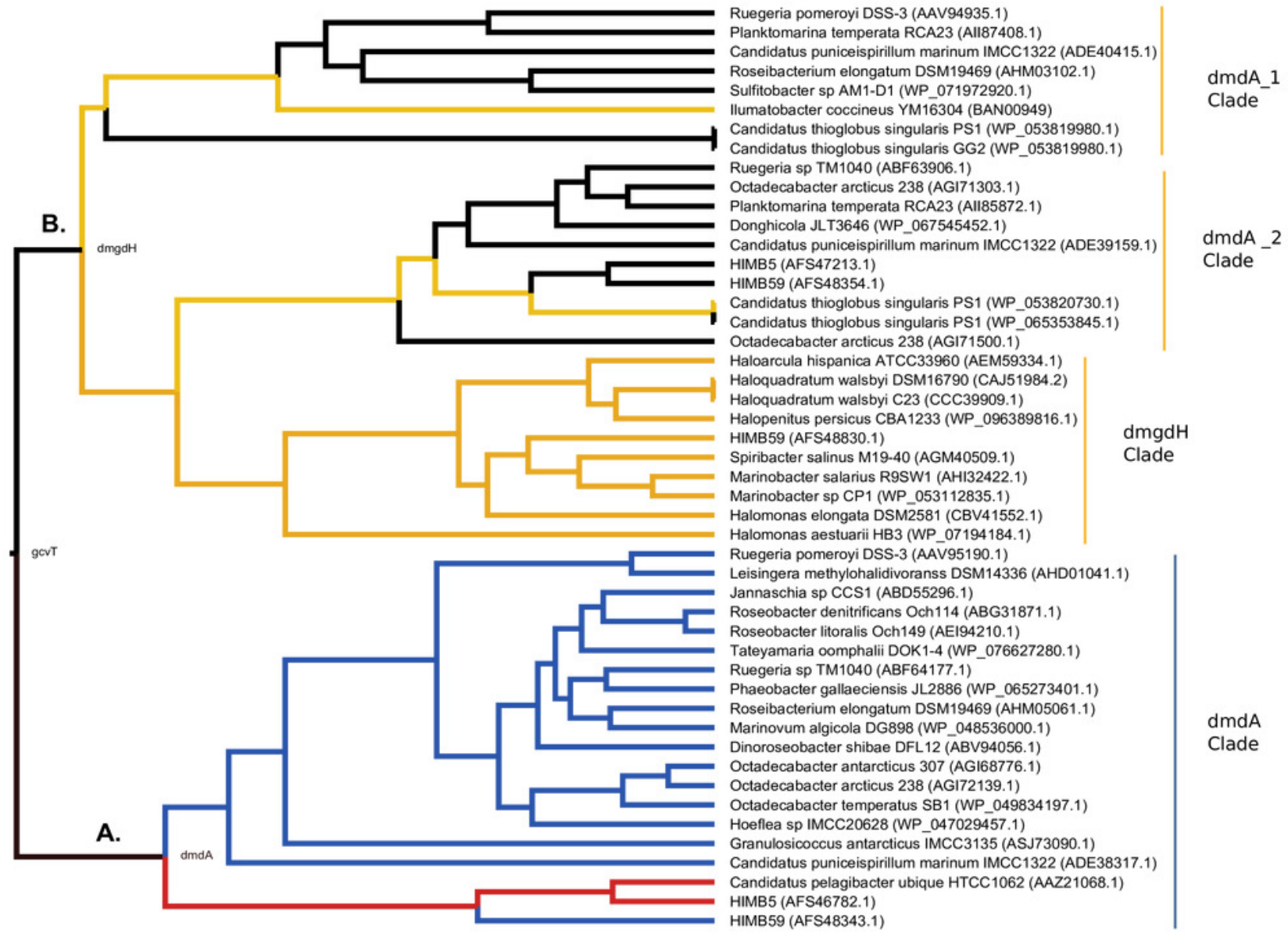

